\title{
Le sujet en langue des signes française
}

Vers une distinction des structures syntaxiques personnelles et impersonnelles

The subject in French Sign Language. Toward a distinction between personal and impersonal syntactic structures.

\section{Myriam Charpentier}

\section{(2) OpenEdition}

Édition électronique

URL : http://journals.openedition.org/tipa/2515

DOI : 10.4000/tipa.2515

ISSN : 2264-7082

Éditeur

Laboratoire Parole et Langage

Référence électronique

Myriam Charpentier, «Le sujet en langue des signes française », TIPA. Travaux interdisciplinaires sur la parole et le langage [En ligne], 34 | 2018, mis en ligne le 18 septembre 2018, consulté le 09 octobre 2020. URL : http://journals.openedition.org/tipa/2515 ; DOI : https://doi.org/10.4000/tipa.2515

Ce document a été généré automatiquement le 9 octobre 2020.

La revue TIPA. Travaux interdisciplinaires sur la parole et le langage est mise à disposition selon les termes de la licence Creative Commons Attribution - Pas d'Utilisation Commerciale - Pas de Modification 4.0 International. 


\title{
Le sujet en langue des signes française
}

Vers une distinction des structures syntaxiques personnelles et impersonnelles

\author{
The subject in French Sign Language. Toward a distinction between personal \\ and impersonal syntactic structures.
}

\section{Myriam Charpentier}

Je remercie mes trois relecteurs anonymes pour leurs observations et questionnements, grâce auxquels j'ai pu retravailler et améliorer la première version de cet article. Je suis également reconnaissante envers mes directrices de recherche, Mmes Anne Carlier, Laurence Meurant et Annie Risler, pour leur relecture attentive et leurs conseils qui m'ont permis d'enrichir ce travail. Les insuffisances qui demeurent sont de mon fait.

Ce média ne peut être affiché ici. Veuillez vous reporter à l'édition en ligne http:// journals.openedition.org/tipa/2515

\section{Introduction}

Langues minoritaires et minorisées, les langues signées (LS) ont été tardivement considérées comme étant des langues à part entière. De ce fait, bien qu'étant des objets d'études riches, ces langues sont encore peu explorées, aussi bien sur le plan linguistique que sur les aspects culturels et sociétaux qui les caractérisent. La présente étude dresse un état des lieux de la recherche sur certaines spécificités du fonctionnement syntaxique des LS, et plus particulièrement concernant les propriétés du sujet syntaxique. La langue des signes française (LSF) sera notre objet d'étude. Toutefois, le déjà-là scientifique, portant aussi bien sur les autres LS que sur les langues vocales, nous servira d'appui. 
3 Notre étude propose une réflexion centrée sur l'identification du sujet syntaxique en LSF, en abordant successivement des constructions personnelles ainsi que des constructions dans lesquelles l'agent est mis en arrière-plan. Pour ce faire, nous nous basons sur un échantillon d'exemples illustrant d'une part la construction canonique, ayant un sujet référentiel, correspondant prototypiquement au rôle d'agent, et d'autre part les constructions qui relèguent à l'arrière-plan l'agent ou l'effacent. Ce travail se présente comme une étude préliminaire et servira de base à une étude de corpus plus vaste, portant sur les mécanismes de mise en arrière-plan de l'agent en LSF.

4 Nous aborderons deux types de constructions syntaxiques reléguant à l'arrière-plan ou effaçant le sujet-agent, i. e. l'argument sélectionné par le prédicat au rôle syntaxique de sujet et correspondant sémantiquement à l'agent, à savoir la construction passive et la construction impersonnelle.

5 Avant de procéder à leur étude, nous nous attarderons sur la construction basique dont elles sont dérivées, à savoir la construction active avec un sujet pleinement référentiel. Comme l'a en effet observé Keenan (1976: 310), cette construction est la structure basique prototypique de la langue et est donc syntaxiquement plus simple que les propositions mettant l'agent en arrière-plan. C'est à partir de cette construction de base que nous dégagerons les propriétés caractéristiques du sujet. Il a été observé que, malgré le statut central du sujet au sein de la proposition (Lazard, 1994 ; Bakker \& Siewierska, 2007), les propriétés qui le caractérisent ne sont pas universelles. En fonction des langues, il peut donc être caractérisé par des propriétés très variables (Keenan, 1976 ; Lazard, 1994), d'ordres syntaxique, morphosyntaxique, sémantique ou pragmatique.

6 Pour ce qui est des constructions mettant l'agent en arrière-plan, celles-ci peuvent correspondre à différentes opérations en fonction des propriétés qui les caractérisent, comme le passif et l'impersonnel. La construction passive est limitée aux verbes transitifs, mettant typiquement en rapport un agent et un patient. Le passif est considéré ici comme un mécanisme qui supprime l'agent de la position argumentale de sujet et qui permet que cette position soit occupée par le patient. Ainsi, à partir d'une phrase transitive active comme (1), on obtient la phrase passive (2) dans laquelle le sujet syntaxique correspond au patient, l'agent pouvant être exprimé par un constituant non argumental prenant la forme d'un complément oblique, ici entre crochets:

(1) Le voleur m'a frappé

(2) L’ai été frappé [par le voleur]

7 Concernant la construction impersonnelle, contrairement à ce que pourrait laisser croire sa dénomination, son analyse ne doit pas mobiliser la notion de "personne", mais se rapporte à l'existence et la nature du sujet (Creissels, 2006). Parmi l'importante hétérogénéité des constructions impersonnelles, nous traiterons ici de la construction caractérisée par une perte de la référentialité du constituant en position sujet (Siewierska, 2011). Cette construction impersonnelle a ainsi les caractéristiques syntaxiques d'une proposition personnelle canonique avec un sujet humain, mais dont la valeur référentielle est indistincte, comme en (3). Nous parlerons ainsi d'une construction à sujet référentiel indistinct : 
(3) On m'a frappé

Si les opérations de mise en arrière-plan de l'agent ont été abondamment étudiées pour les langues vocales, elles le sont encore marginalement pour les LS. Une telle étude nécessite une bonne connaissance $d u$ fonctionnement des relations syntaxiques en LS. Or, dans les études de ces langues, la fonction de sujet est généralement associée d'office au constituant porteur du rôle sémantique d'agent, sans que la dimension syntaxique de ce constituant soit considérée. Les opérations de mise en retrait de l'agent en (2) et (3) montrent toutefois que le sujet syntaxique n'est pas nécessairement l'agent, mais peut être le patient. Nous examinerons si l'hypothèse d'une noncorrélation systématique entre fonction syntaxique de sujet et rôle thématique de l'agent se confirme dans les cas de constructions de mise en arrière-plan de l'agent en LSF, tels que le passif et l'impersonnel.

9 Dans la section 2.1, nous présentons différents critères d'identification du sujet qui ont été définis dans la littérature. La section 2.2 souligne les importantes particularités des LS, également à prendre en compte dans cette analyse. La section 2.3 introduit deux types d'analyse de la langue des signes, attribuant la fonction syntaxique de sujet à différentes unités significatives du discours : au point de départ du mouvement verbal ou à la place du corps du signeur. Après la section 3, décrivant la méthodologie suivie, la section 4 est centrée sur une analyse de corpus et sur les observations qui peuvent en être tirées : différents types de constructions personnelles et impersonnelles sont alors identifiées, sous un angle comparatif. La section 4.1 est consacrée aux constructions personnelles à sujet exprimé et présente une analyse de la position du constituant sujet dans une proposition personnelle basique en LSF, permettant de décrire certaines propriétés de l'argument sujet. Dans la section 4.2 sont présentées et analysées des constructions personnelles de notre corpus, dans lesquelles le sujet n'est pas instancié. À partir de cette base de recherche, la section 4.3 décrit les spécificités de quelques procédés de mise en arrière-plan de l'agent en LSF présents dans notre corpus. Cette démarche de comparaison entre constructions personnelles et constructions dans lesquelles l'agent est mis en retrait servira d'introduction à une analyse future des différentes structures de mise en arrière-plan de l'agent attestées dans cette langue.

\section{Etat de l'art}

\subsection{Quelques critères d'identification du sujet syntaxique dans les langues vocales}

Plusieurs critères formels d'identification du sujet syntaxique prototypique sont donnés dans la littérature sur les langues vocales. Ils permettent de distinguer l'argument sujet, parmi les différents constituants qui participent à la construction de la phrase (Creissels, 2006; Bakker \& Siewierska, 2007). Ces critères peuvent être regroupés en trois grandes catégories.

- Les propriétés morphologiques. En fonction des langues, ces propriétés peuvent, par exemple, correspondre au marquage casuel, ou encore à l'accord du verbe avec son sujet ; 
- Les propriétés syntaxiques. Peuvent notamment être retenues, en fonction des langues, des contraintes positionnelles, la présence obligatoire du sujet ou encore la contrainte de coréférence avec les réfléchis et réciproques ;

- Les propriétés sémantiques. De manière générale, dans les langues, le sujet syntaxique prototypique a tendance à être un agent, hautement référentiel et caractérisé par les traits [+animé] et [+humain].

11 En français, par exemple, dans une phrase canonique comportant une forme verbale finie, le sujet peut être repéré morphologiquement par le fait qu'il régit l'accord verbal. Du point de vue de la syntaxe, il peut être identifié par sa position linéaire : l'ordre de base des constituants étant SVO (Sujet - Verbe - Objet), parmi les constituants nominaux qui participent à la construction de la phrase, l'argument en position préverbale est, par défaut, le sujet. Par ailleurs, en présence d'une forme verbale, le sujet est en principe obligatoirement exprimé, mais il peut toutefois être omis auprès $\mathrm{du}$ deuxième prédicat verbal dans une construction de coordination de deux propositions (Lazard, 1994, 2009): Pierre rit et (ø/il) applaudit la scène. Parmi les propriétés syntaxiques du sujet en français, on note également des propriétés coréférentielles. Un pronom réfléchi est nécessairement coréférentiel avec le sujet: Pierre se lave. Enfin, sur le plan de la syntaxe, le sujet prototypique a tendance à être agentif et présente un haut degré de référentialité, comme en (1). La contrainte de l'agentivité peut néanmoins facilement être transgressée en français : J'ai reçu un coup dans la figure, alors que celle du haut degré de référentialité semble plus robuste:? De l'huile bouillante a brûlé le voleur, ? Du gibier est dans la forêt.

\subsection{Quelques particularités des LS à prendre en compte pour identifier le sujet en LSF}

12 Avant d'examiner si les critères précédents sont opératoires pour identifier le sujet en LSF, il convient de prendre en compte certaines caractéristiques des LS, dont le point commun est de s'exprimer à travers les canaux visuels et gestuels. Cette modalité particulière a d'importantes répercussions sur les ressources exploitables grammaticalement. Elle implique notamment la motivation des signes linguistiques et la spatialisation des relations syntaxiques (Cuxac, 1993 et 2000 ; Miller et al., 1999; Millet, 2006). En effet, la modalité visuelle et gestuelle des langues signées induit certaines spécificités sur les plans morphologique et syntaxique, qui peuvent notamment jouer un rôle quant aux propriétés de la position sujet et de son expression.

\subsubsection{Le choix du cadre théorique}

En France, les premiers travaux sur la langue des signes se sont fortement appuyés sur cette question de la modalité visuelle et gestuelle et sur son impact dans la langue. C'est à partir des travaux de Cuxac que s'est développé le modèle dit sémiologique (Cuxac, 2000 ; Sallandre 2001, 2003 ; Boutet et al., 2010 ; Garcia, 2010 ; Garcia \& Martinod, 2017). À travers ce modèle, la description de la langue distingue deux formes du dire : celles qui disent - les signes conventionnels - et celles qui ont la particularité de dire en donnant à voir - appelées les transferts. Le modèle sémiologique a gagné une grande influence dans le champ de la linguistique de la LSF. En décrivant les structures de transfert comme étant des formes du dire, le parti pris de cette approche consiste à 
considérer la langue des signes française en fonction de sa prédisposition iconique, et à l'opposer de ce fait aux langues vocales. Il en découle, selon Cuxac, l'inadéquation des outils conceptuels développés par la linguistique des langues vocales pour décrire les langues signées. Notre démarche a un autre objet. Elle vise à identifier les spécificités syntaxiques de la LSF, et se fonde dès lors sur un essai de mise à l'épreuve des concepts syntaxiques établis en linguistique sur le cas particulier d'une langue signée. L'emprunt d'outils élaborés pour décrire le fonctionnement des langues non-signées nous permettra d'établir par la suite des comparaisons, utiles notamment en vue de la création d'outils d'évaluation et de modèles d'acquisition. C'est pourquoi nous abordons cette étude de la LSF avec une orientation typologique. Cette prise de position est fondée sur le fait que le langage, qu'il s'exprime par des signes vocaux ou par la modalité visuo-gestuelle, est indissociable de la cognition, et qu'il peut donc être intéressant et éclairant de comparer les structurations que véhiculent les différents types de systèmes linguistiques, tout en dégageant leurs différences. Ainsi, bien que nous restions vigilante vis-à-vis de l'application aux langues signées des terminologies développées dans l'étude des langues vocales et que nous gardions à l'esprit la variabilité linguistique, nous restons ouverte au recours de ces terminologies et proposons un discours de coopération et de dialogue avec les études sur les langues vocales.

\subsubsection{Quelques particularités du fonctionnement morphologique des LS}

Le premier préalable est de distinguer certaines catégories grammaticales, notamment le nom et le verbe. L'identification de ces catégories a fait l'objet d'analyses pour plusieurs LS. Il a souvent été affirmé que l'opposition entre la catégorie nominale et la catégorie verbale n'est pas lexicale, parce qu'un même signe peut être utilisé comme nom ou comme verbe. Or, certaines marques morphologiques et phonologiques peuvent permettre de lever l'ambiguïté et d'identifier la nature verbale ou nominale d'un signe, telles que l'amplitude, la vitesse ou la réduplication de celui-ci, ou encore la manière de mouvement qu'il traduit. C'est notamment le cas pour la langue des signes américaine (ASL) ou la langue des signes australienne (Auslan) (Supalla \& Newport, 1978 : 94 ; Schembri et al., $2002: 26$ ).

15 En LSF, l'absence d'opposition catégorielle lexicale a également été observée (Cuxac, 2000 ; Risler 2007). Comme dans d'autres LS (Johnston, 2001), l'ambiguïté est toutefois souvent levée par le co-texte ou l'environnement syntaxique immédiat. De plus, comme en ASL ou en Auslan, l'analyse de certaines paires nom-verbe en LSF a mis en évidence des critères morphologiques permettant de distinguer ces deux catégories grammaticales. Risler $(2002,2007)$ distingue deux types de signes, en fonction de marquages morphologiques et du rôle des constituants dans la construction syntaxique : les signes figés (les constituants nominaux) et les relateurs (les prédicats).

16 Les signes nominaux sont définis selon cette analyse comme étant non marqués morphologiquement, accompagnés d'un regard adressé à l'interlocuteur, et réalisés de manière stable dans l'espace, $i$. e. ils ne construisent pas les relations syntaxiques (Risler, 2007 : 94). Dans la présente étude, nous ne retiendrons que ce dernier critère de non-construction des relations syntaxiques. En effet, le critère de la stabilité morphologique des signes nominaux est discutable, puisque l'on ne peut pas parler d'invariabilité. Tout comme les verbes, les noms peuvent porter des indices de pluriel. On retient notamment le procédé du balayage de l'espace, i. e. le déplacement du signe 
sur un emplacement. Ce procédé, que l'on retrouve également parmi les marquages morphologiques de la catégorie verbale, est le marquage du pluriel le plus fréquent concernant les noms en LSF (Millet, 2008).

Quant aux signes prédicatifs, leur fonction de mise en relation syntaxique des constituants permet de les distinguer des constituants nominaux. Porteurs de marquages spatiaux, manuels ou non-manuels (Bahan et al., 2000), les signes prédicatifs marquent spatialement les relations syntaxiques (Risler, 2007 : 94).

Ce rôle de l'espace dans la morphologie verbale et la construction des relations syntaxiques permet notamment de prendre conscience de l'impact de la modalité visuo-gestuelle sur le système des LS. En effet, en langue des signes, certains espaces se voient attribuer une valeur référentielle. Ces emplacements sémantisés, ou loci, qui renvoient par le mécanisme de l'anaphore aux arguments, jouent un rôle important dans la flexion verbale. Ce procédé de sémantisation de l'espace repose de manière très visible sur les articulateurs manuels, sans oublier le rôle crucial des articulateurs nonmanuels, comme l'orientation du buste, du regard ou encore la mimique faciale et les mouvements de la tête (Lillo-Martin, 1986 ; Bahan et al., 2000 ; Meurant, 2008). Comme pour l'analyse de signes nominaux, le regard est une nouvelle fois à prendre en compte puisque, orienté vers les mains ou l'un des marquages spatiaux, il accompagne la construction des relations syntaxiques. Ainsi, réactivés par différents articulateurs, les loci renvoient spatialement à l'argument qui leur a été référentiellement associé (Risler, 2013).

La spatialité jouant un rôle important dans la flexion verbale, nous nous attarderons sur la question de l'encodage du sujet par certains loci lors de l'analyse de nos exemples.

\subsubsection{Contraintes positionnelles en LS}

20 Après avoir établi la distinction entre la catégorie nominale et la catégorie verbale, et mis en valeur le rôle de la spatialisation des relations syntaxiques, abordons la question de la position du constituant sujet dans la proposition et vis-à-vis du verbe. Du point de vue typologique, le sujet est généralement l'élément de la proposition le plus à gauche (Keenan, 1976) et tend donc à se trouver en position préverbale. En effet, un échantillon de 1497 langues recensées par The World Atlas on Language Structure (Dryer, 2013) met en évidence que le sujet est placé de manière préférentielle avant le verbe, soit dans $80 \%$ des langues examinées. C'est donc l'une des hypothèses que nous soumettons à examen dans la présente étude.

21 L'identification des fonctions syntaxiques à partir de l'ordre canonique des constituants est difficile à appliquer aux langues signées (Lillo-Martin, 1986). En effet, au sein de la communauté scientifique, les avis divergent au sujet de l'ordre des constituants et restent pour la majorité à l'état d'hypothèses. Il n'en reste pas moins que l'ordre des constituants en LSF ne serait pas complètement libre (De Langhe et al., 2003). Des structures instanciant les ordres SOV (Sujet - Objet - Verbe), SVO ou encore OSV, ont notamment pu être identifiées, OSV étant pourtant très rare parmi les langues vocales du monde (Tomlin, 1986 : 22). De manière générale, c'est l'ordre SOV qui est considéré comme préférentiel en LSF (Yau, 1993; De Langhe et al., 2003; Amblard \& Voisin, 2008). Toutefois, cette question de l'ordre préférentiel des constituants en LSF mériterait une recherche plus approfondie. C'est pourquoi, dans la présente étude, 
nous ne mettrons pas l'accent sur cet aspect du fonctionnement syntaxique de la langue, mais que nous retiendrons, à partir des trois ordres observés (SVO, OSV et $\mathrm{OSV}$ ), que le constituant sujet occupe la position préverbale.

\subsection{Deux approches sur le sujet en LS}

La question de l'agent est centrale dans notre étude des propriétés du sujet en LSF. Le sujet correspond-il systématiquement à l'agent, comme cela a pu être considéré dans certains travaux?

Afin de laisser de côté l'aspect sémantique et de nous concentrer sur une définition syntaxique du sujet en LSF, nous cherchons les paramètres qui permettent d'identifier l'emplacement qui encode le sujet. Celui-ci peut-il être encodé par n'importe quel locus ou n'est-il, au contraire, spatialisé que sur un type d'emplacement précis? Correspondil au point de départ du mouvement verbal ? Le type de verbe employé peut-il nous aider à identifier l'emplacement porteur de la fonction sujet ?

Pour répondre à ces interrogations, deux perspectives de recherche sur le marquage de la fonction sujet en LS peuvent nous guider. Dans un premier temps, avec la section 2.3.1, nous nous penchons sur les analyses de verbes directionnels, i. e. dont le mouvement est réversible. Avec ces verbes, les points de départ et d'arrivée du mouvement verbal intègrent la référence aux actants et sont associés à des rôles sémantiques précis. Le mouvement d'un verbe comme dire, par exemple, active d'abord la référence à l'agent. Le point d'arrivée correspond donc au patient. À l'inverse, d'autres verbes directionnels, comme inviter, intègrent initialement l'emplacement correspondant au patient (Meir et al., 2007). L'interprétation de différents emplois de verbes directionnels peut, notamment, nous renseigner sur l'assignation des fonctions syntaxiques aux différents emplacements sémantisés. Dans un deuxième temps, en 2.3.2, nous soulignerons le rôle que d'autres recherches ont attribué à la place du corps du signeur dans l'encodage des fonctions syntaxiques.

\subsubsection{Le point de départ du mouvement verbal porteur de la fonction sujet}

Les études sur les LS se sont souvent intéressées aux signes prédicatifs : à la flexion verbale (Bahan et al., 2000 ; MacLaughlin et al., 2000; Cormier, 2014), ou encore à une classification des verbes (Padden, 1988; Engberg-Pedersen, 1993). La structure prédicative fait, en général, l'objet d'une analyse sémantique et les rôles thématiques centraux d'agent et de patient sont clairement identifiés (Cuxac, 2003 ; Millet, 2004). En revanche, la description des fonctions syntaxiques est rarement donnée. En observant les interprétations d'emplois de verbes directionnels dans les études sur la LSF, nous avons cependant constaté que, majoritairement, ces interprétations donnent lieu à des phrases actives. Le point de départ du mouvement verbal - qui renvoie à l'agent du procès - encode la fonction sujet. Par conséquent, le point d'arrivée du mouvement verbal - qui correspond au patient - porte la fonction de l'objet. C'est notamment ce que montre la Figure 1, avec ses diverses réalisations du verbe donner : 
Fig. 1 Analyse du fonctionnement des verbes directionnels de la grammaire IVT (Moody, 1998 : 159)

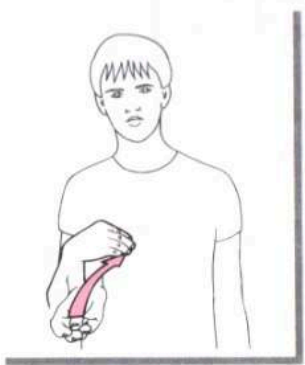

TU-ME-DONNES

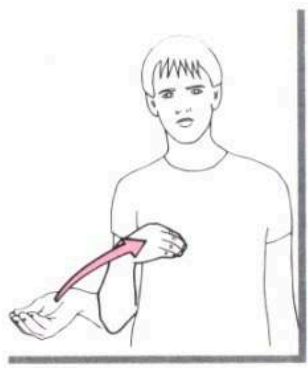

IL-ME-DONNE

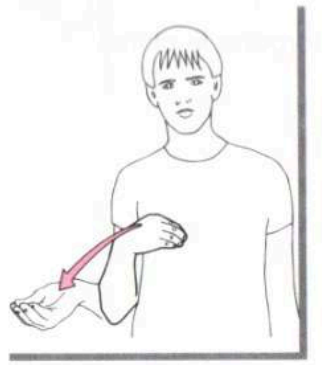

JE-LUI-DONNE



IL-TE-DONNE

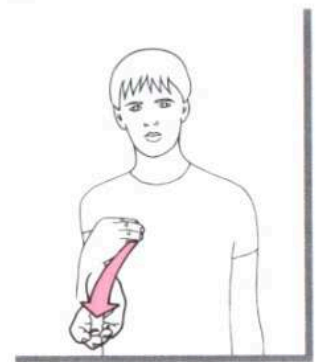

JE-TE-DONNE

Les six images de la Figure 1 illustrent parfaitement la réversibilité des signes directionnels en LSF, mais également le choix systématique de la perspective active qui a pu être fait dans chaque traduction des exemples, malgré l'inversion du mouvement verbal d'une image à l'autre : pour chaque cas, le point de départ du mouvement verbal, qui intègre la référence à l'agent, est interprété comme encodant la fonction sujet.

Guitteny (2005) s'intéresse à cette réversibilité du mouvement verbal et à son impact sur les fonctions syntaxiques. Au niveau sémantique, la particularité des verbes directionnels est que l'agent est toujours présent dans la construction verbale. En effet, avec ce type de verbes, la référence aux actants est activée par le mouvement verbal. Ainsi, si des indicateurs des arguments participent à la flexion verbale, l'agent peut-il être mis en retrait dans la construction? Avec ces verbes réversibles, comment distinguer la voix passive de la voix active ? À ce sujet, Guitteny (2005) propose une distinction entre passif et inverse :

- un cas d'inverse explicite référentiellement l'agent. De plus, au niveau informationnel, l'inversion du mouvement verbal n'implique aucun changement. Il ne s'agit que d'un changement d'orientation du point de vue, depuis celui de l'agent explicité. Par conséquent, l'emplacement renvoyant à l'agent, activé par le point de départ du mouvement verbal, encoderait la fonction sujet. C'est notamment le cas des exemples TU-ME-DonNES ${ } \mathrm{ou}_{\text {IL-ME-DONNE' }}$ dans la Figure 1;

- un cas de passif, au contraire, sera identifiable grâce à la perte de référentialité de l'agent, par exemple, du fait d'une spatialisation vague. L'agent étant mis en retrait, la perspective du patient est mise en valeur et l'emplacement associé à ce dernier encode la fonction sujet. Si l'on revient à l'exemple ${ }_{\text {il-me-donne }}$ de la Figure 1 , en proposant par exemple, que le point de départ du mouvement verbal (porteur du rôle thématique de l'agent) ne s'est vu attribuer aucune valeur sémantique, l'absence de référentialité de cet espace nous amènerait à identifier un cas de passif. Nous pourrions alors suggérer l'interprétation suivante : Il m'a été donné [...]. 

l'hypothèse que, parallèlement à ce qui se passe dans les langues vocales, en LS, des composantes sémantiques particulières du verbe sont lexicalisées par des unités spécifiques (Talmy, 1985). Ainsi, avec certaines formes verbales, le corps encoderait le sujet, tandis que les articulateurs manuels renverraient aux autres arguments. À travers leur proposition, Meir et al. (2007) reprennent la classification des verbes proposée par Padden (1988), qui définit trois catégories de verbes en fonction des possibilités de marquage argumental qui les caractérisent. Toutefois, Meir et al. analysent quant à eux ces verbes en fonction du rôle qui peut être attribué au corps du signeur dans l'encodage des fonctions syntaxiques :

- Les verbes neutres (plain verbs). Ces verbes qui comportent un ancrage corporel, i. e. dont le mouvement se réalise au niveau de l'une des parties du buste du signeur, tels manger ou penser, sont caractérisés par l'assignation de la fonction de sujet à la place du corps du signeur. La marque du sujet ne correspond pas nécessairement à la première personne.

- Les verbes à accord / verbes directionnels (agreements verbs). Avec ce type de verbes, parmi lesquels dire ou informer, la place du corps du signeur ne se voit pas forcément attribuer la fonction sujet. En revanche, elle correspond systématiquement à la première personne.

- Les verbes de mouvement / de déplacement (spatial verbs). Ce troisième type de verbes sera laissé de côté dans cette étude. En effet, ces verbes n'impliquant pas le corps du signeur comme emplacement de réalisation du signe, l'analyse du corps comme sujet n'a plus lieu d'être.

Ainsi, selon cette hypothèse, la place du corps du signeur encode le sujet avec certains types de verbes en particulier: systématiquement avec les verbes neutres mais de manière moins systématique avec les verbes directionnels. Cette fonction syntaxique 
serait donc associée à certains emplacements en fonction du type de verbes auquel nous avons affaire. Bien qu'ils s'appuient sur l'ASL et l'ISL (langue des signes israélienne), Meir et al. (2007) formulent l'hypothèse que cette analyse pourrait être appliquée à d'autres LS.

Dans cette recherche, nous nous en tenons à une conception syntaxique du sujet que l'on distinguera donc de l'agent. Au regard de ces différentes prises en compte du corps du signeur dans la littérature, plusieurs types d'analyse se présentent à nous. À travers l'étude des exemples tirés de notre corpus, nous tentons dans un premier temps de comparer ces hypothèses, afin de déterminer si la fonction de sujet est attribuée à certains emplacements en particulier en LSF. Dans quelle mesure devons-nous interpréter le point de départ du mouvement du verbe ou le corps du signeur comme référant à l'argument sujet? Afin de répondre à cette question, cette étude analyse différents emplois de verbes à ancrage corporel et de verbes directionnels tirés de notre corpus. Cette démarche d'analyse des comportements syntaxiques avec ces deux types de verbes nous amène également à nous demander s'il existe un lien entre l'emplacement encodant le sujet et le type de verbes employé. Par ailleurs, dans le cas de verbes réversibles, l'encodage du sujet par le point de départ ou d'arrivée du mouvement verbal engendre-t-il un changement informationnel ?

Grâce à l'observation de nos exemples, nous tenterons également de définir si, en LSF, un ordre préférentiel des constituants régit la position du sujet par rapport au verbe. De plus, notre étude cherche à déterminer si l'instanciation du sujet est obligatoire en LSF, ou si cette langue accepte des structures dans lesquelles le sujet n'est pas instancié lexicalement. À la lumière de notre cheminement, nous tâcherons dans un deuxième temps de déterminer si des comportements syntaxiques permettent de distinguer les constructions personnelles et celles mettant en arrière-plan l'agent.

\section{Méthodologie}

Les résultats de cette étude exploratoire s'appuient sur des données empiriques, analysées d'après une approche fonctionnelle-typologique. Notre corpus rassemble des données semi-spontanées, produites en contexte de monologue narratif. Ces données sont extraites de deux corpus : LS-COLIN ${ }^{1}$ (2002) et Finitestory ${ }^{2}$ (2013).

Le corpus LS-COLIN (Langues des Signes - Cognition, Linguistique, INformatique) résulte d'un projet d'étude pluridisciplinaire, réalisé entre 2000 et 2002. Il regroupe les productions de plusieurs locuteurs sourds de la LSF. Dans la présente étude, nous avons exclusivement et exhaustivement utilisé les récits portant sur le passage à l'Euro et les attentats du 11 septembre 2001 .

Le corpus Finitestory a été élaboré plus récemment dans le cadre d'une recherche de Master en 2013. Il se fonde sur la méthodologie de Dimroth et al. (2010), conçue à l'origine pour l'étude linguistique des langues vocales. Le corpus regroupe les productions de deux locuteurs sourds de la LSF, qui s'expriment chacun à partir de trente clips vidéo très brefs, immédiatement après leur visionnage. Ces clips, liés entre eux, mettent en scène trois personnages dans un même environnement, et constituent une histoire complète. Les personnages se différencient par la couleur de leurs vêtements. Les locuteurs se servent ainsi de ces couleurs pour y faire référence. La 
couleur devenant l'identité du personnage, ils parlent ainsi du «bleu», du «vert » et $\mathrm{du}$ « rouge », pour renvoyer aux personnages correspondants.

Ces corpus permettent de mettre en évidence certains phénomènes universels des langues: la construction de références et l'anaphore. De plus, le corpus Finitestory, élicitant des énoncés très brefs, rend aisée la recherche de références personnelles et de sujets syntaxiques canoniques.

L'analyse s'est effectuée en deux étapes :

Dans un premier temps, nous avons repéré et analysé dans les deux corpus les structures personnelles, afin d'établir les propriétés de sujets canoniques.

Dans un deuxième temps, à partir du corpus LS-COLIN, nous nous sommes consacrée à la recherche et à l'analyse de constructions dans lesquelles l'agent est mis en retrait, $i$. $e$. non spécifié ou non référentiel. Ces deux étapes nous ont permis d'établir une comparaison entre les structures personnelles et impersonnelles et d'en identifier les propriétés respectives.

40 Nous rappelons à ce stade que nos résultats proviennent d'une recherche préliminaire. En effet, les constructions de mise en arrière-plan de l'agent présentées dans cet article correspondent à quelques cas de figure fréquents dans notre corpus, sans prétention à l'exhaustivité. Il est entendu que ce travail introduit une étude de corpus de plus grande ampleur, qui offrira une plus grande variété de données, ainsi que des résultats quantitatifs.

\section{Les propriétés de l'argument sujet en LSF}

Les sections 4.1 et 4.2 traitent de différentes structures personnelles attestées en LSF, et permettent d'établir une base de recherche autour de constructions personnelles basiques de cette langue. Les propriétés du sujet décrites dans ces sections servent de support pour la comparaison avec les constructions impersonnelles, abordées dans la section 4.3. Cette démarche comparative a permis de repérer les similitudes et divergences entre ces deux types de constructions référentielles.

\subsection{L'expression du sujet dans des constructions personnelles}

Pour illustrer notre analyse, voici tout d'abord en (4) et (5) des structures personnelles, tirées du corpus Finitestory.

(4) BLEU + PENSER + QUOI FAIRE

('le personnage bleu pense : que faire?’) 


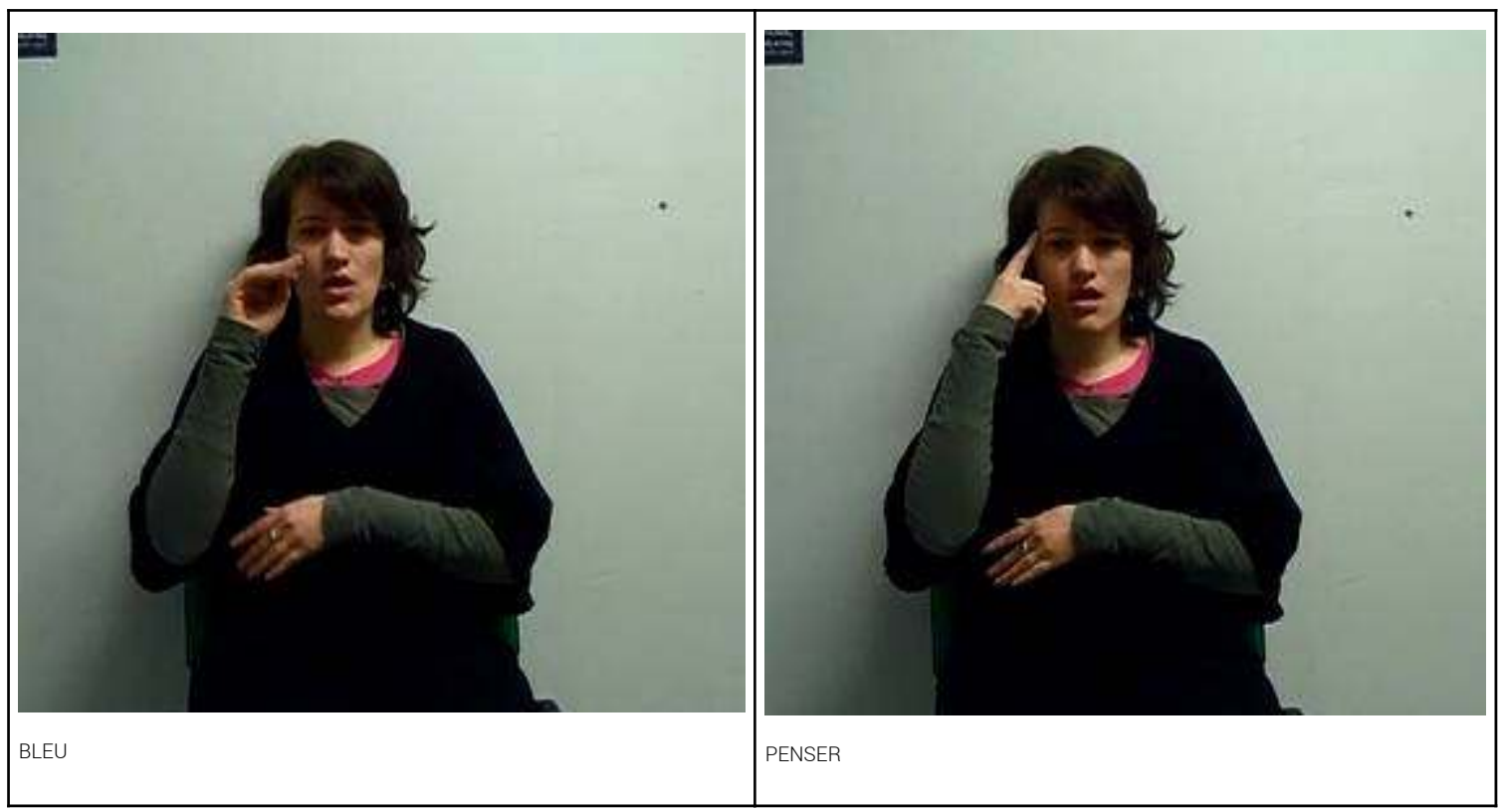

(5) CHAMBRE + VERT + LUI (pointage anaphorique sur un emplacement) + SE RÉVEILLER ('dans la chambre verte // dans la chambre du vert, lui il se réveille.')

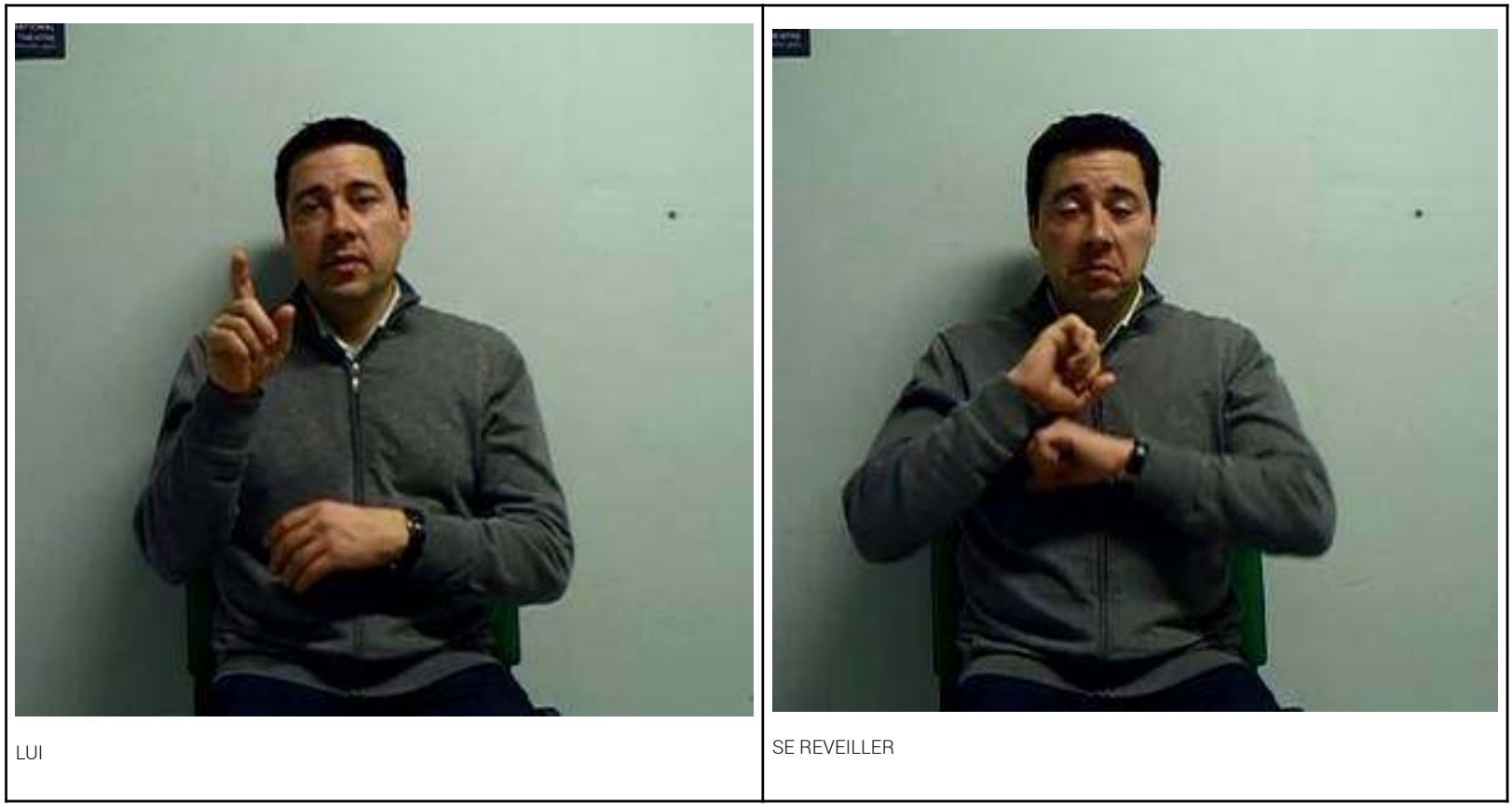

43 Dans l'exemple (4), le locuteur introduit la pensée d'un personnage, au discours direct. C'est la séquence qui précède l'extrait de discours rapporté qui nous intéresse ici, i.e. le constituant nominal et le prédicat avec lequel il est en relation : BLEU + PENSER, que nous avons traduit par 'le bleu pense'. Cette interprétation, attribuant le rôle syntaxique de sujet au constituant BLEU, est soutenue par plusieurs caractéristiques de l'énoncé.

44 Pour commencer, l'orientation du regard du signeur vers son interlocuteur (ici, la caméra) nous permet d'identifier BLEU comme un constituant nominal. Au contraire, 
une rupture du regard accompagne le prédicat penser à ancrage corporel. Notons alors que l'unique constituant nominal est en tête de phrase et précède le verbe. Nous formulons ainsi l'hypothèse qu'il s'agit de l'argument sujet du prédicat penser.

De plus, au niveau énonciatif, nous observons en (4) une caractéristique majeure des LS : la place importante du corps du signeur dans les procédés référentiels. À un niveau énonciatif particulier, appelé la prise de rôle, le corps du signeur ne réfère plus au signeur lui-même, mais occupe la place de l'argument sujet (Risler, 2011). Cette possibilité d'encodage du sujet à la place du corps du signeur renforce ainsi le statut du sujet en tant qu'élément central de la phrase (Lazard, 1994 ; Bakker \& Siewierska, 2007). Les procès, effectués ou subis par le protagoniste, sont alors exprimés par le locuteur du point de vue interne du personnage. En (4), le changement d'ancrage référentiel est annoncé par une nette rupture du regard, accompagnant la fin de la réalisation du constituant nominal et le début de réalisation du prédicat. La place du corps du signeur ne fait donc plus référence au locuteur, sujet énonciateur, mais à une tierce personne.

En suivant l'hypothèse de Meir et al. (2007), nous proposons d'analyser la place du corps du signeur comme encodant la fonction de sujet du verbe à ancrage corporel penser.

$\mathrm{Au}$ niveau sémantique, il y aurait coréférentialité entre la valeur référentielle du constituant nominal bleu et celle du corps du signeur. Cette proposition d'analyse coïncide d'ailleurs avec la valeur référentielle du corps du signeur dans le discours direct, au niveau énonciatif de la prise de rôle.

L'exemple (5) illustre un autre cas de figure. Tout d'abord, le regard adressé à l'interlocuteur (de nouveau, le locuteur s'adresse à la caméra) nous permet d'identifier un premier groupe de deux signes nominaux CHAMBRE + VERT, suivi d'un pointage pronominal. Nous avons interprété ce groupe comme un circonstant spatial, permettant d'identifier un ancrage spatio-temporel spécifique. Grâce à la prise en compte de cet ancrage, nous pouvons analyser le pointage comme un indice de troisième personne, référant au personnage localisé précédemment dans la chambre dite " verte " (ou « du [personnage] vert »). Après le pointage pronominal, une rupture du regard accompagne la réalisation du prédicat se réveiller avec lequel est en relation la référence personnelle.

L'exemple (5) illustre ainsi plusieurs phénomènes intéressants. D'une part, la place importante de la spatialisation en LSF est constatée, puisque ce mécanisme permet ici de déterminer l'ancrage référentiel et de faire référence à une entité par un pointage vers l'emplacement qui lui est associé. D'autre part, avec ce pointage pronominal, nous observons, une fois de plus, que le sujet précède le verbe. Pour finir, l'emploi de ce verbe à ancrage corporel nous amène à nouveau à nous interroger sur le rôle de la place du corps du signeur. L'investissement du corps dans l'expression du procès pourrait être un indicateur de l'encodage du sujet par la forme verbale (Meir et al., 2007). Dans la suite de notre analyse, nous utiliserons le terme de locus corporel pour référer à cet indicateur spatial marqué par le corps du signeur.

Les énoncés (4) et (5) sont tirés du corpus Finitestory et ont permis d'illustrer le rôle du corps du signeur dans l'encodage de la fonction de sujet. La suite de l'analyse sera, quant à elle, illustrée par des extraits du corpus LS-Colin. Voyons à présent le rôle de la spatialisation au travers de l'exemple (6) : 
(6) TOI (pointage sur un emplacement devant le signeur) + DEMANDER (l'action demander part de ce même emplacement et va vers le signeur) + AVIS (localisation sur le corps du signeur)

('toi, tu me demandes mon avis')

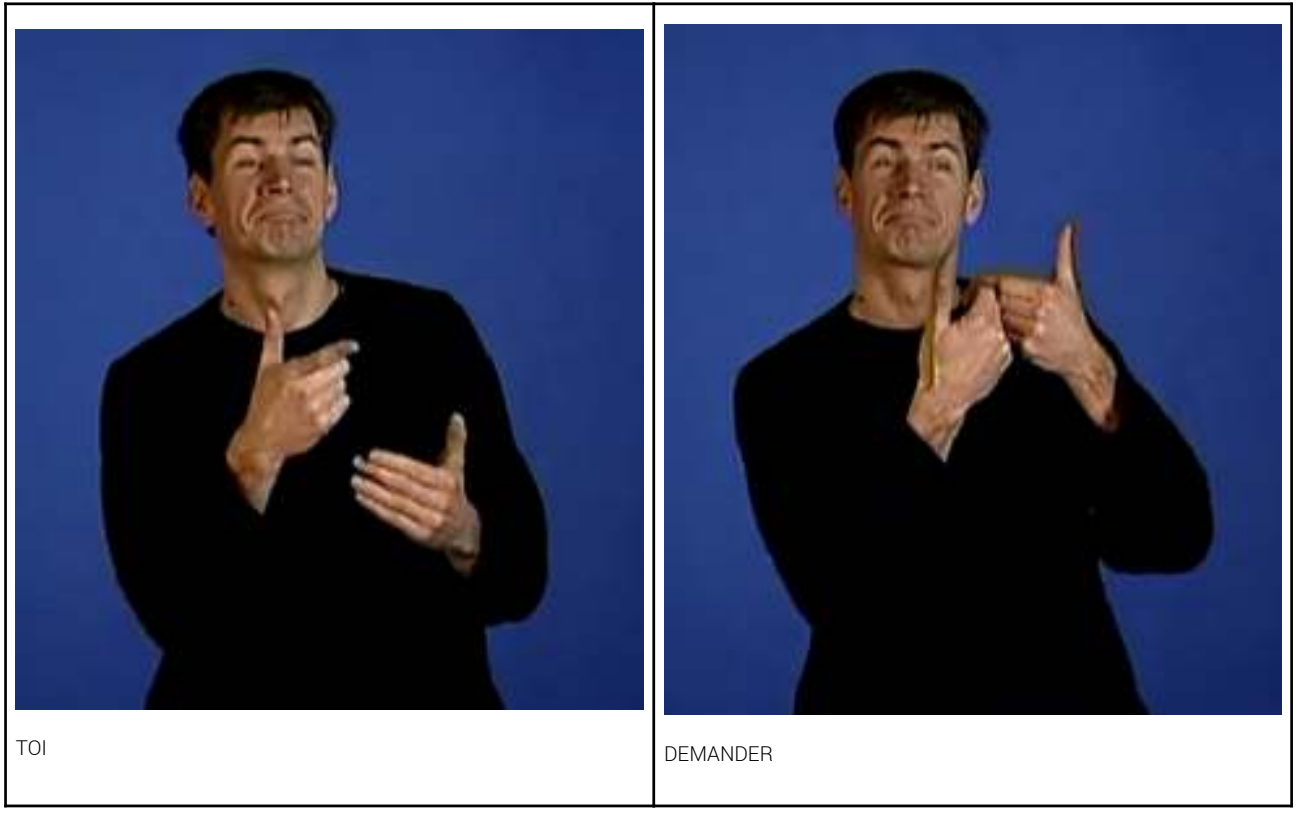

Contrairement aux exemples (4) et (5) pour lesquels il s'agit d'emploi de verbes neutres, i. e. dont la forme ne change pas, l'exemple (6) illustre l'emploi d'un verbe réversible. Le signe verbal DEMANDER peut se réaliser en partant du corps du signeur vers un emplacement, ou comme ici, depuis un emplacement vers le corps du signeur. Le point de départ du mouvement verbal, ici l'emplacement devant le signeur, porte le rôle sémantique d'agent, nous parlerons du locus 1 . Comme le montre la première illustration de l'exemple, un pointage pronominal, également orienté vers le locus 1, précède le prédicat. Le regard même du signeur est orienté vers cet emplacement, tout au long de l'énoncé.

Dans cet exemple, nous proposons dans un premier temps d'analyser le point de départ du mouvement verbal comme encodant la fonction sujet. Nous faisons ainsi l'hypothèse que cet emploi du verbe demander serait un cas d'inverse (Guitteny, 2005). Pour comprendre cette interprétation, soulignons que le signeur s'adresse à une personne physique, présente dans la situation d'énonciation : l'individu qui vient de lui indiquer le thème sur lequel il doit s'exprimer. L'agent du procès, dont la référence est spatialisée sur le locus 1, est donc parfaitement explicite. De plus, la mise en relief de cet agent, par le pointage pronominal qui précède, suggère une interprétation active : il y aurait ainsi corrélation entre rôle thématique d'agent et fonction syntaxique de sujet dans cet exemple.

Nous pourrions cependant proposer une seconde interprétation, attribuant comme en (4) et (5) la fonction sujet à la place du corps du signeur. L'interprétation de cet énoncé serait alors tout autre, puisque nous obtiendrions ainsi, soit une lecture passive (' 
Il m'est demandé par toi ...'), soit une lecture nominale ('De toi je reçois la demande...'). Avec ces deux propositions d'interprétation, la place du corps du signeur retrouve une place centrale dans la construction.

Pour conclure, tout comme nous l'avons constaté avec les exemples (4) et (5), nous observons en (6) que le constituant nominal qui précède directement le verbe pourrait se voir attribuer la fonction syntaxique de sujet. À partir de ces observations, nous pourrions faire l'hypothèse qu'en LSF, le sujet est en position préverbale.

Cependant, au regard des emplois de verbes à ancrage corporel en (4) et (5), nous nous sommes également interrogée sur le rôle du corps du signeur dans la langue. En suivant Meir et al. (2007), nous avons fait l'hypothèse qu'avec ces verbes, le locus corporel participait à la flexion verbale en marquant spatialement l'unique argument, i. e. le sujet. Cette hypothèse, attribuant à la place du corps du signeur la particularité d'encoder la fonction sujet, correspond également à la seconde interprétation de l'exemple (6). Cela nous amène notamment à formuler l'hypothèse selon laquelle la place du corps du signeur marquerait la fonction syntaxique de sujet, quel que soit le type de verbe employé. Nous tenterons d'enrichir cette hypothèse dans la suite de notre analyse et ainsi de répondre à la question suivante : dans quelle mesure devonsnous interpréter le point de départ du mouvement du verbe ou le corps du signeur comme référant à l'argument sujet ? Cette question nous amènera également à revenir sur l'ordre des constituants.

\subsection{Le paramètre de l'argument nul}

Dans notre recherche des propriétés du sujet en LSF, la question de la présence même de cet argument est importante. Certaines études sur d'autres LS, comme l'ASL ou la langue des signes russe (RSL), font en effet l'hypothèse de structures à argument nul (Lillo-Martin, 1986; Bahan et al., 2000 ; Kimmelman, 2017). La LSF accepte-t-elle, elle aussi, des constructions dans lesquelles le sujet est non-instancié ? Répondre à cette question nous permettra notamment de mieux comprendre le fonctionnement du sujet syntaxique en LSF.

La syntaxe de certaines langues accepte que les positions argumentales du sujet ou de l'objet soient vides. C'est le cas du japonais, qui permet l'omission du sujet et de l'objet, mais aussi de la majorité des langues romanes, où le paramètre de l'argument nul concerne uniquement le sujet. Dans ces langues, malgré l'absence de constituant instancié dans l'une de ces positions, d'autres procédés permettent de récupérer la référence. Prenons l'exemple de l'italien, dont la flexion verbale permet d'interpréter la référence au sujet non exprimé. Entre le français et l'italien, on observe ainsi :

\begin{tabular}{|l|l|}
\hline français & italien \\
\hline Je parle & Parlo \\
\hline Tu dors & Dormi \\
\hline Il travaille & Lavora \\
\hline
\end{tabular}


Alors que l'omission du constituant sujet est possible en italien grâce à la flexion verbale, on constate que le français impose la présence d'un constituant en position de sujet. Ce phénomène explique notamment la présence obligatoire d'un sujet pronominal explétif - $i$. e. vide sémantiquement - dans certaines structures syntaxiques. C'est ce que l'on observe notamment dans les constructions verbales passives, où l'argument externe a été supprimé (7), ou avec les verbes sans argument externe (8) :

\begin{tabular}{|l|l|l|}
\hline (7) & *a été volé beaucoup de bijoux & Il a été volé beaucoup de bijoux \\
\hline$(8)$ & *pleut & Il pleut \\
\hline & *semble que le bar a fermé & Il semble que le bar a fermé \\
\hline
\end{tabular}

Alors qu'en français on constate que la langue impose la présence d'un constituant en position de sujet, notre étude de corpus indique que des constructions dans lesquelles le sujet n'est pas exprimé sont attestées en LSF. S'agit-il de constructions à argument sujet nul ? Le paramètre de l'argument sujet nul n'étant pas homogène d'une langue à l'autre, l'analyse suivante des exemples (9), (10) et (11) en définit les propriétés :

(9) POLICE + ÉQUIPE + F-B-I + CHERCHER (l'action chercher est signée de manière ample) // Ø ACCUSER + LUI (pointage sur un emplacement à droite) + HOMME + PERSONNE + NOM + B-E-N L-A-D-E-N + BEN LADEN (nom signé)

('la police et l'équipe du FBI ont beaucoup cherché ; ils ont accusé un homme, une personne du nom de Ben Laden, signé "comme ça" ')

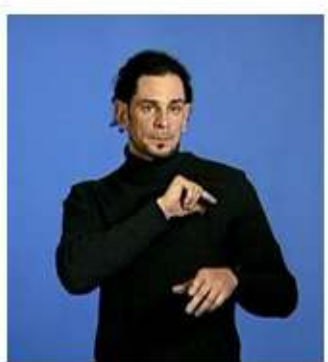

POLICE

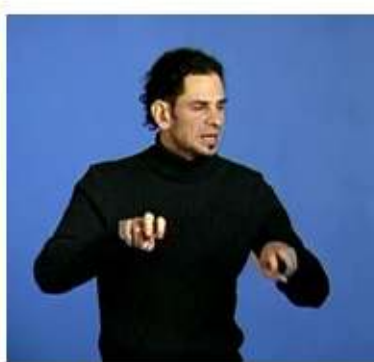

CHERCHER

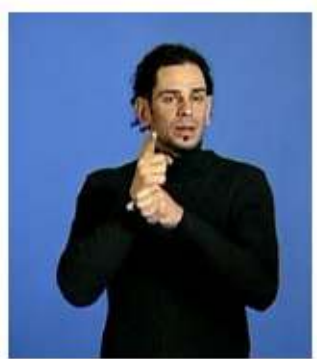

ACCUSER

59

La compréhension de l'exemple (9) reposant sur le contexte linguistique, nous avons choisi de représenter la structure sans sujet exprimé ACCUSER + LUI, avec l'énoncé qui la précède. Dans ce premier énoncé, l'investissement du corps du signeur, lors de la réalisation du signe prédicatif CHERCHER, indique que celui-ci est produit en prise de rôle. La place du corps du signeur marque l'emplacement associé à l'argument sujet (Risler, 2011). Notons que le locus corporel reprend anaphoriquement la référence introduite par le groupe de signes nominaux, en position préverbale : POLICE + ÉQUIPE + FB-I. Nous pouvons en déduire, qu'en (9), le sujet du premier énoncé est instancié lexicalement, puis marqué par la place du corps du signeur en prise de rôle. La structure qui nous intéresse est produite juste après une nette rupture du regard: le 
signeur, sorti de la prise de rôle, emploie le verbe accuser. À la suite du prédicat, le patient est instancié nominalement puis localisé sur un espace à droite du signeur, par un pointage de l'index. En revanche, aucun constituant nominal ne précède immédiatement le prédicat, et ne permet d'identifier l'agent. Dans cette situation comment identifier le sujet syntaxique ? Prenons en compte le contexte linguistique et notamment la proposition qui précède. Sur le plan discursif, l'enchaînement des deux propositions constitue un cas de continuité thématique. Nous pouvons ainsi faire l'hypothèse qu'en LSF, comme en français, le sujet peut être omis dans des propositions coordonnées (Lazard, 1994). La fonction de sujet serait alors portée par le constituant POLICE, en tête du premier énoncé. Malgré l'absence d'instanciation du sujet dans la seconde proposition, le sujet a été exprimé précédemment et la référence est donc récupérable.

Un phénomène similaire est observable dans l'exemple (10):

(10) FILS + PROFITER + INFORMER (l'action informer va de l'emplacement associé à fils, vers le signeur)

('j’ai profité d'avoir été informé par mon fils' ou 'je profite que mon fils m’ait informé')

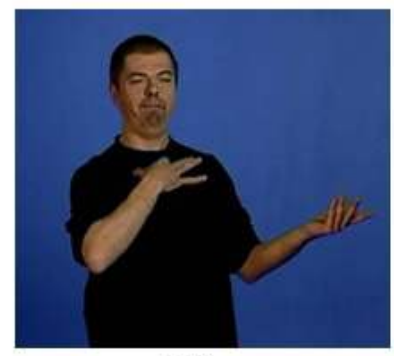

FILS



PROFITER

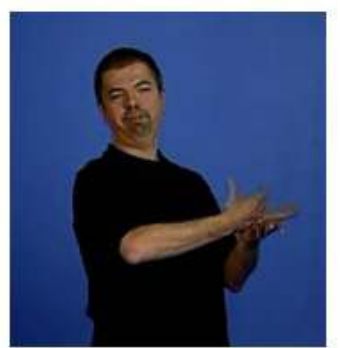

INFORMER

61 En (10), on constate qu'un seul argument est explicité en tête de phrase, par le signe nominal FILs. Toutefois, nous pouvons compter sur le contexte linguistique pour identifier le second: le signeur lui-même. En effet, précédemment, celui-ci exprime qu'il a informé certaines personnes de l'université, sur le moyen de se procurer des euros. Dans l'énoncé suivant, que nous analysons ici, il indique comment il a lui-même été mis au courant : grâce à son fils. Non seulement l'interprétation est facilitée grâce au contexte linguistique proche, mais il s'agit également d'une reprise de ce qui a été exprimé au début du discours. Ce contexte permet d'identifier l'agent du verbe profiter : le signeur lui-même. Au contraire, il est bénéficiaire du procès informer, le statut d'agent correspondant alors au fils.

Avec l'expression du verbe à ancrage corporel profiter, nous pouvons adopter l'hypothèse de Meir et al. (2007), et proposer d'analyser l'emplacement auquel est associé le rôle thématique de l'agent, $i$. e. la place du corps du signeur, comme marquant la fonction sujet. Cela illustre à nouveau la place centrale du corps dans la langue. Néanmoins, la suite de l'énoncé questionne cette hypothèse.

En effet, nous avons ensuite affaire au verbe informer. À l'instar du verbe demander analysé en (6), il s'agit d'un verbe réversible dont l'orientation est inversée, i. e. orientée vers le corps du signeur. Le statut de bénéficiaire est aisément identifiable, 
marqué par le locus corporel, qui réfère dans cet ancrage énonciatif au signeur luimême. Quant à l'agent, il est explicité au début de l'énoncé par le constituant nominal fils. En outre, dans le même temps, la référence a été spatialisée, sur un emplacement à gauche du signeur : nous parlerons du locus 2. Le point de départ du mouvement verbal intègre ainsi la référence à cet agent.

Puisque nous avons identifié les rôles sémantiques des arguments de cet exemple (10), qu'en est-il des fonctions syntaxiques ? Deux interprétations se présentent.

La première amènerait à identifier un cas d'inverse. En effet, la mise en relief de la référence au fils, par le signe nominal fILS qui introduit l'énoncé et le maintien de la main gauche sur le locus 2, tout au long de l'énoncé, nous conduit à interpréter ce locus 2 comme porteur de la fonction de premier argument. Le rôle d'agent et la fonction de sujet étant encodée par le même emplacement (le point de départ du mouvement verbal), nous serions dans une perspective active, et donc bien face à un cas d'inverse. Nous aurions donc une interprétation du type : je profite que mon fils m'ait informé. Au vu de l'agencement de la proposition, rappelant un phénomène de parenthèse, cette analyse permet également de comprendre la place du verbe profiter, entre le verbe informer et son agent fils. Afin d'être plus fidèle à la structure originelle de la phrase et notamment à la topicalisation, nous pourrions ainsi proposer : mon fils, j'en ai profité, il m'avait informé.

Cependant, une seconde interprétation, prenant en compte la place du corps du signeur, correspondrait à une perspective passive. Notamment, au regard de l'hypothèse de Meir et al. (2007), un indicateur de la fonction rôle syntaxique du sujet serait intégré au verbe profiter par la place du corps du signeur. Ce marquage, qui rend la référence au sujet hautement accessible (Ariel, 2001), nous amène à faire l'hypothèse d'une continuité thématique entre les structures prédicatives. De ce fait, le sujet des prédicats profiter et informer serait identique et serait donc, à chaque fois, associé à la place du corps du signeur. Concernant le verbe informer, le corps du signeur aurait alors le statut de patient. De ce fait, cet emploi d'un verbe réversible n'illustrerait pas un cas d'inverse mais de construction passive (Guitteny, 2005), que nous pourrions traduire par j'ai profité d'avoir été informé par mon fils.

Le rôle syntaxique de la place du corps du signeur peut à nouveau être étudié avec l'exemple (11) :

(11) JOUR + ONZE + SPTEMBRE + 2001 // ce jour-là (pointage localisé devant le signeur) + JOUR + FORT + SE RAPPELER

('le jour du 11 septembre 2001 ; ça, c'était un jour fort je m'en rappelle') 




SE RAPPELER

Cet énoncé est caractérisé par l'absence d'explicitation de l'argument sujet. S'agissant du début du discours du locuteur, ainsi que de l'emploi d'un verbe à ancrage corporel, la référence à l'actant n'est ni disponible dans le contexte, ni récupérable grâce à la forme verbale. De nouveau, cela appuie l'hypothèse selon laquelle la LSF accepte des structures dans lesquelles le premier argument n'est pas instancié. Dans ce cas, comment identifier le sujet du prédicat se rappeler?

Pour interpréter cet exemple, nous nous sommes, tout d'abord, aidée du contexte extralinguistique. S'agissant d'une tâche de narration, dans laquelle les locuteurs doivent donner leur avis sur l'attaque contre le World Trade Center, nous avons fait le choix d'interpréter la place du corps du signeur comme marquant à la fois la fonction de sujet et la première personne. Ainsi, selon cette hypothèse, le signeur fait référence à lui-même, dans la situation hic et nunc de l'énonciation. De plus, comme nous avons pu le dire précédemment, il a longtemps été considéré par les études sur la LSF que, lorsque le sujet de l'énoncé est également le sujet de l'énonciation, la référence à la première personne est souvent implicite (Cuxac, 2000 ; Morgenstern et al., 2016), et le signeur n'a alors pas besoin d'expliciter cette référence par un signe d'auto-désignation (Morgenstern, 1997). La référence au sujet peut être interprétée grâce au contexte et à l'ancrage dans la situation d'énonciation (Risler, 2011).

70 En somme, dans cet énoncé, nous pouvons faire l'hypothèse que le signeur fait référence à lui-même. Avec le verbe à ancrage corporel se rappeler, la place du corps du signeur encode la fonction de sujet (Meir et al., 2007) et correspondrait à la première personne.

71 Cependant, une seconde analyse est possible pour cet exemple et nous amène à réfléchir sur la valeur de première personne du corps du signeur. En effet, en (11), aucun indice ne nous permet d'identifier référentiellement l'agent. De ce fait, nous pouvons supposer que cette absence totale d'indice référentiel correspond à une mise en arrière-plan de l'agent. La structure syntaxique de l'énoncé implique ainsi une interprétation arbitraire, d'autant que, dans la suite directe du discours, le locuteur indique que le monde était choqué, et réfère ainsi à une masse générale d'individus. 
72 Dans cet exemple, deux composants non-manuels appuient l'hypothèse de la prise de rôle : le regard et l'investissement du buste. En effet, c'est à partir de la rupture du regard, qui suit le signe nominal JouR, qu'est investi le corps du signeur. Le locus corporel se verrait alors associer une valeur référentielle indistincte: un trait [+humain] mais [-spécifié]. Cela introduit le changement de prosodie et l'accentuation des signes FORT et SE RAPPELER. À partir de cette seconde analyse, nous pourrions ainsi proposer une interprétation de l'exemple telle que : ça, c'était un jour fort, on s'en rappelle.

73 Ainsi, malgré la mise en arrière-plan de l'agent du fait de sa faible référentialité, l'encodage de la fonction sujet par le corps du signeur illustre une nouvelle fois la place centrale du sujet dans la langue.

74 À travers les exemples de la section 4.2, nous avons observé d'une part que la LSF accepte des constructions dans lesquelles le sujet n'est pas instancié lexicalement. Toutefois, pouvons-nous vraiment parler de construction à argument sujet nul, comme le font certaines études sur d'autres LS (Lillo-Martin, 1986; Bahan et al., 2000; Kimmelman, 2017) ? Bahan et al. (2000), défendent qu'en ASL les constructions à argument nul sont rendues possibles grâce à la morphologie verbale : quel que soit le type de verbe, des indices, manuels et/ou non-manuels, intégrés aux formes verbales permettent l'identification des arguments. Nos exemples montrent que la LSF accepte elle aussi des constructions dans lesquelles l'argument sujet est non-instancié lexicalement, mais identifiable grâce à la morphologie verbale. Toutefois, l'analyse que nous en proposons est différente : selon notre hypothèse la non-instanciation du sujet ne correspond, non pas au paramètre de l'argument sujet nul, mais à une expression de l'anaphore. Le rôle des loci, i.e. des indices spatiaux des arguments, a été décrit dans la section 2.2.1 et, dans cette section, nous avons pu observer leur fonctionnement dans l'analyse des exemples (9) et (10). Les loci étant porteurs de la valeur syntaxique des arguments, l'activation de ces emplacements par la flexion verbale conduit alors selon nous à un procédé d'anaphore. Ainsi, malgré le fait que le sujet ne soit pas systématiquement instancié lexicalement, le locus intégré à la forme verbale instancie spatialement la référence à celui-ci.

D'autre part, l'exemple (11) a montré que, lorsque le sujet n'est pas instancié lexicalement et ne peut être identifié sémantiquement, il prend une valeur générique. En outre, nous avons constaté que l'hypothèse de Meir et al. (2007), associant la fonction de sujet à la place du corps du signeur, demeure valide lorsque le sujet n'est pas identifiable sur le plan sémantique. La fonction de sujet peut ainsi être encodée par le corps du signeur quelle que soit sa valeur référentielle.

76 De plus, l'observation des exemples (9), (10) et (11) a apporté des éléments nouveaux à notre étude. Dans un premier temps, nous avons constaté avec (9) que, dans deux propositions coordonnées, le sujet du prédicat, omis dans la deuxième proposition, peut être récupéré à partir de la première. Il s'agit d'ailleurs d'un comportement syntaxique que l'on trouve également en français (Lazard, 1994). Ainsi, en plus d'être interprétable grâce aux différents indices, manuels et/ou non-manuels, présents dans l'énoncé, la référence au sujet est récupérable dans le contexte linguistique.

77 Par ailleurs, parallèlement à notre questionnement lors de l'analyse de l'exemple (6), l'exemple (10) nous a amenée à nous interroger sur l'interprétation du point de départ du mouvement verbal, comme étant porteur de la fonction sujet. À nouveau, nous avons mis cette réflexion en regard avec Meir et al. (2007), portant sur la place du corps du signeur dans la forme verbale. En effet, lorsque le locus corporel porte la référence 
au patient, assigner la fonction de sujet soit à la place du corps du signeur, soit au point de départ du mouvement verbal, peut opposer deux interprétations de l'énoncé : une lecture passive ou une lecture active.

De même, en (11), nous nous sommes intéressée à la valeur du corps du signeur, en contexte de mise en arrière-plan de l'agent. Le verbe à ancrage corporel amène l'encodage du seul argument - le sujet - par la place du corps du signeur. L'absence d'indice référentiel nous permettant d'identifier l'agent a conduit à faire l'hypothèse que la place du corps du signeur pourrait se voir assigner la fonction de sujet, indifféremment de son degré de référentialité. La partie suivante pourra nous aider à préciser notre analyse des structures mettant l'agent en arrière-plan.

\subsection{L'expression du sujet et la mise en arrière-plan de l'agent}

Les sections 4.1 et 4.2 nous ont permis d'analyser divers types de constructions personnelles prototypiques en LSF et d'en proposer différentes interprétations. Ces possibilités d'analyses nous ont conduite à formuler sous forme d'hypothèses ce que pourraient être certaines propriétés caractérisant le sujet en LSF. Nous avons notamment tenté de démontrer que l'instanciation lexicale du sujet n'est pas obligatoire dans cette langue. De plus, à travers l'analyse de nos exemples, nous formulons l'hypothèse que la place du corps du signeur encode la fonction de sujet quel que soit le type de verbe employé. Le marquage du sujet serait ainsi réalisé sur un type de locus particulier : le locus corporel.

À présent, nous allons procéder à l'examen des énoncés (12) à (15), dans lesquels l'agent est mis en retrait, afin de dégager leurs propriétés distinctives :

(12) PERSONNE (réalisé de manière tronquée) + REGARDER (l'action regarder se fait sur une localisation haute, à droite)

('les gens regardaient le ciel')



TIPA. Travaux interdisciplinaires sur la parole et le langage, 34 | 2018 
(13) PERSONNE + CHOC + FUIR (l'action de fuir se réalise de manière diffuse) // POUSSIERE + PLEIN + FUIR (l'action de fuir se réalise de manière diffuse)

('les gens étaient choqués ils fuyaient ; il y avait plein de poussière, ils fuyaient')

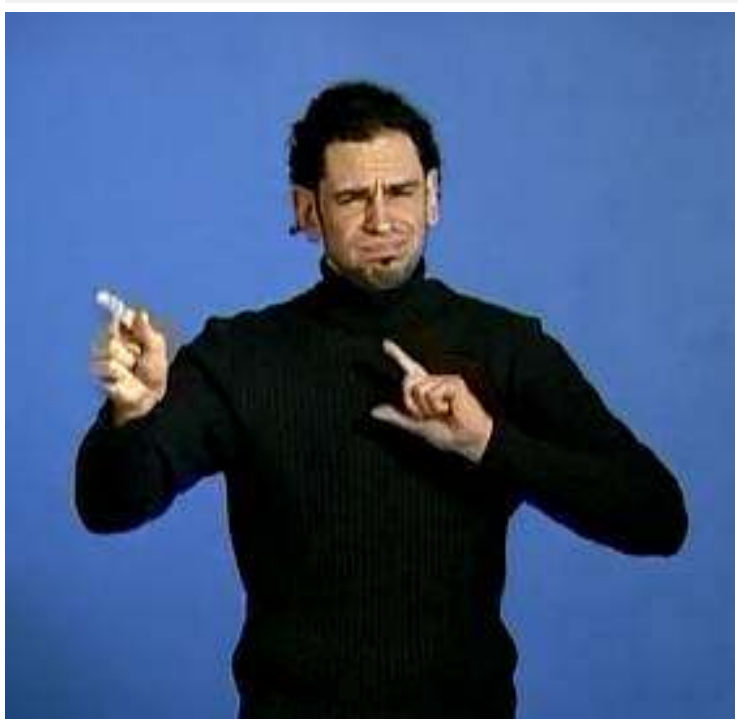

FUIR

(14) AVANT + PREMIER + JANVIER + INFORMER (l'action informer va d'un emplacement en haut à gauche du signeur vers le signeur lui-même) + euro

('avant le $1^{\mathrm{er}}$ janvier, on m'a informé sur l'Euro')

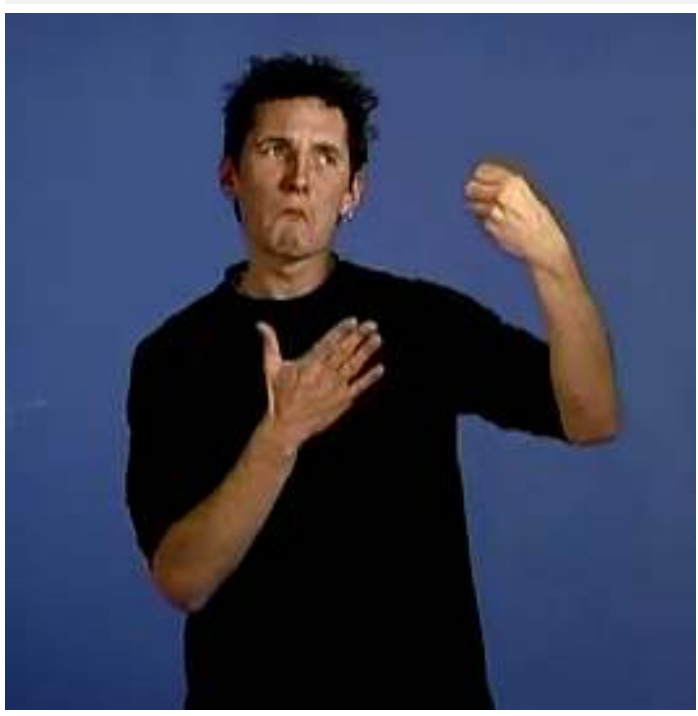

INFORMER

81 Dans un premier temps, on observe en (12) un emploi du prédicat regarder. Au regard $\mathrm{du}$ mouvement verbal nous constatons que le rôle thématique d'agent est associé au locus corporel. Le patient est quant à lui spatialisé sur un espace très haut devant le signeur. Grâce à l'investissement du corps du signeur, nous pouvons également déduire que ce prédicat est marqué par une prise de rôle. Le corps joue ainsi le rôle de 
marqueur de la fonction de premier argument (Meir et al., 2007 ; Risler, 2011), et la valeur référentielle qui lui est associée est indiquée par l'indice de prise de rôle qui précède : le signe PERSONNE réalisé de manière tronquée. En effet, si les autopointages sont des indices de prise de rôle très fréquents, ils ne sont pas les seuls à pouvoir introduire un changement d'ancrage énonciatif (Risler, 2016). Ici, l'indice de prise de rôle permet d'identifier la valeur référentielle du corps du signeur, et, par la même occasion, le sujet-agent du verbe regarder.

Dans l'identification du sujet, le rôle des articulateurs manuels est également à prendre en compte. En effet, comme le montre la deuxième illustration de l'exemple, la configuration manuelle apporte un marquage morphologique et encode la pluralité de l'agent.

À présent que la structure de l'exemple a été décrite, revenons à ce qui nous motive pour l'analyser comme une construction impersonnelle. Cette hypothèse repose sur l'indice de prise de rôle, i. e. le signe tronqué PERSONNE, en lien avec le prédicat bimanuel. En effet, le sujet-agent, qui n'a jamais été introduit par un constituant lexicalement plein, est marqué par l'indice de prise de rôle et la place du corps du signeur. Mais, bien que ces indices réfèrent à un animé pluriel et portent plus précisément le trait [+humain], ils sont référentiellement faibles. Le groupe d'individus n'étant pas spécifié, nous pouvons conclure que nous avons affaire ici à une construction référentielle, de forme impersonnelle (Siewierska, 2008 et 2011). Ainsi avec ce verbe directionnel, l'agent, associé à un emplacement, ne peut pas être effacé, mais il est malgré tout laissé dans le flou dans cet exemple, mettant l'accent sur le procès.

L'exemple (13) illustre un autre type de procédé de mise en retrait de l'agent. Comme plus haut, pour certaines structures personnelles de notre analyse, nous pouvons constater en (13) que, lors de la deuxième réalisation du verbe fuir, le sujet n'est pas exprimé. Toutefois, comme évoqué plus haut également, la référence au sujet est récupérable dans les contextes linguistique et extralinguistique: elle est introduite précédemment par le constituant nominal personne, en tête de phrase. De plus, le verbe étant marqué par la prise de rôle, la fonction sujet est également encodée par la place du corps du signeur (Risler, 2011).

En (13), l'agencement des propositions peut nous renseigner sur cette construction n'instanciant pas le sujet. La répétition du prédicat dans l'énoncé est nettement visible. Notons que les deux prédicats sont séparés par le groupe de signes nominaux POUSSIÈRE + PLEIN, que nous avons traduit par il y a plein de poussière. Nous pouvons observer ici un phénomène de parenthèse, caractérisé par une construction en écho (Blondel \& Le Gac, 2007). Encadrée par deux occurrences du même prédicat fuir, cette partie de l'énoncé permet au signeur de faire un commentaire sur l'événement décrit. Ainsi, en (13), les prédicats des deux propositions, séparées par la parenthèse, ont le même sujet-agent. Le partage du sujet entre deux prédicats de propositions coordonnées a déjà été analysé dans notre étude, avec les énoncés personnels (9) et (10).

À présent que l'agencement des propositions dans l'exemple a été clarifié, revenons vers son interprétation impersonnelle. Nous avons noté que la fonction de sujet-agent du verbe fuir était portée par le constituant nominal personne, en tête de phrase. Comme dans l'exemple (12) cet agent est [+humain], mais réfère à des individus non- 
spécifiés. Cette faible référentialité du constituant sujet nous amène ainsi à proposer qu'il s'agirait ici d'une construction à sujet référentiel indistinct.

Une fois de plus, sur le plan syntaxique nous ne sommes pas en mesure de différencier une construction personnelle d'une construction impersonnelle, puisque les deux types de constructions sont caractérisés par les mêmes propriétés. C'est uniquement sur le plan sémantique que nous constatons que la référentialité du constituant est indistincte.

Pour finir, l'exemple (14) illustre un phénomène syntaxique décrit en langue des signes catalane (LSC) (Barberà \& Quer, 2013). Comme en (6) et en (10), l'exemple (14) contient un verbe réversible, dont les arguments sont spatialisés. Le rôle de patient est encodé par le locus corporel et correspond référentiellement au signeur. En revanche, l'agent, marqué sur un espace non-sémantisé, n'est ni référentiel, ni récupérable dans le contexte.

Pour ce qui est de l'espace de signation, nous constatons que celui-ci est particulier. Activé par le regard, puis par le mouvement verbal, l'espace associé à l'agent est très haut: au niveau de la tête du signeur. Il s'agit d'un usage spécifique du procédé de spatialisation qui demande à revenir aux espaces définis par Millet (2004 et 2006). Dans son étude, elle distingue en effet cinq types d'espaces, parmi lesquels l'espace dit de l'agent indéfini. Tout comme nous l'observons en (14), Millet situe cet espace à hauteur des tempes, de chaque côté de la tête du signeur. Ainsi, comme l'ont constaté Barberà \& Quer (2013) en LSC, l'absence de référentialité de l'agent peut jouer sur la morphologie spatiale des verbes réversibles en LSF et provoquer la spatialisation de l'agent sur un locus latéral haut. Par ailleurs, la faible référentialité de l'agent et sa spatialisation particulière peuvent conduire à s'interroger sur la fonction syntaxique qu'il encode. L'exemple (14) correspond-il à une perspective active ou passive? Malgré sa faible référentialité, la mise en valeur de l'agent par sa spatialisation particulière nous amène à faire l'hypothèse d'une perspective active. L'analyse de l'exemple suivant peut nous guider dans notre cheminement.

Nous constatons dans notre corpus que l'emploi d'un emplacement latéral haut, en cas d'utilisation d'un verbe réversible dont l'agent est non-référentiel, n'est pas systématique, comme l'illustre l'exemple (15):

(15) ORGANISER + ORGANISER (les actions organiser sont exprimées amplement avec un balayage de droite à gauche devant le signeur) // INFORMER + INFORMER (les actions informer, exprimées avec un mouvement ample, vont de plusieurs emplacements devant le signeur, vers plusieurs emplacements au niveau du buste du signeur)

('on s'organise ; les uns les autres sont informés') 


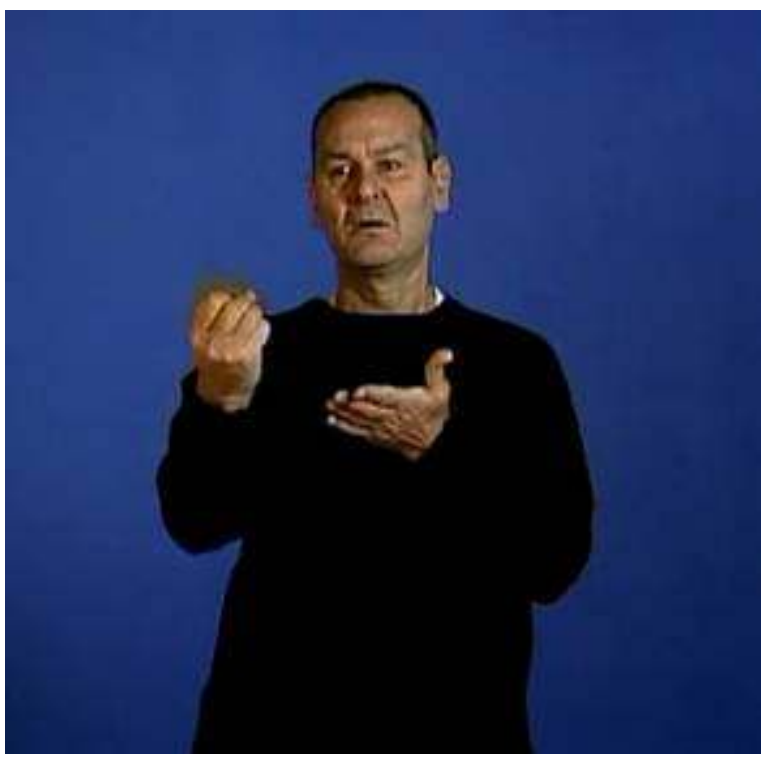

INFORMER

91 Comme on peut le constater grâce à la description et l'illustration de l'exemple, en (15), les locus intégrés par le mouvement du verbe directionnel informer ne sont porteurs d'aucune valeur référentielle. Qu'il s'agisse de l'emplacement renvoyant à l'agent ou celui correspondant au patient, ils n'ont pas été sémantisés au préalable. Cette fois cependant, les espaces ne sont pas situés au niveau de la tête du signeur, mais devant lui et à hauteur d'épaule. Cette faible référentialité est caractérisée par plusieurs paramètres, dont, en premier lieu, la réduplication du signe verbal. En LSF, la réduplication du verbe est fréquente et peut marquer la pluralité (Millet, 2008). Ici, cette notion est renforcée par un balayage de l'espace, à la fois par le regard et les mouvements verbaux, lors de la réduplication des deux prédicats organiser et informer.

92 Si les énoncés (14) et (15), illustrant deux emplois du même verbe, correspondent tous deux à une mise en arrière-plan de l'agent, en revanche, ils ne sont pas caractérisés par les mêmes propriétés syntaxiques. En effet, au vu des deux procédés de spatialisation de l'agent utilisés, nous pouvons faire l'hypothèse que ces énoncés relèvent de deux mécanismes de mise en arrière-plan de l'agent différents :

- en (14), nous avons formulé l'hypothèse que l'utilisation du locus latéral haut traduise une mise en valeur de la perspective de l'agent et ainsi l'assignation de la fonction de sujet à ce locus particulier. Le sujet syntaxique étant l'agent, il s'agirait ainsi d'une perspective active. Peut-il s'agir alors d'un cas d'inverse? La faible référentialité de l'agent invalide d'office cette proposition et nous amène à formuler une autre hypothèse, selon laquelle l'opération de mise en arrière-plan de l'agent que nous observons en (14) serait un cas de construction impersonnelle (Siewierska, 2011).

- en (15), un faible degré de référentialité caractérise les deux arguments. Le mouvement verbal, rédupliqué et diffus, nous amène à faire l'hypothèse que l'agent comme le bénéficiaire pourraient être assimilés à une masse d'individus plutôt qu'à un seul. L'agent étant mis en retrait par cette spatialisation vague sur des espaces non-sémantisés met en valeur la perspective du patient. Cette analyse conduit à proposer une interprétation de cet énoncé avec une perspective passive. L'emplacement associé au patient, i. e. le corps du signeur, encode la fonction sujet. 
Pour conclure, la comparaison des derniers énoncés permet de faire l'hypothèse qu'en LSF, cette différence caractérisant la spatialisation de l'agent dépend du mécanisme de mise en arrière-plan à l'œuvre dans la proposition.

\section{Conclusion} ifier notre hypothèse concernant la non-corrélation systématique entre la fonction syntaxique de sujet et le rôle thématique de l'agent. Partant de cette observation, nous nous sommes tournée vers les caractéristiques morphologiques de la flexion verbale et du marquage du sujet. L'intérêt majeur de nos résultats figure à ce stade dans un démenti de l'hypothèse de Meir et al. (2007) que nous formulons dans nos analyses. En effet, alors que leur proposition distingue le marquage du sujet en fonction de deux types de verbes - les verbes neutres et les verbes à accord - nous défendons au contraire une analyse équivalente de ces verbes. En effet, nous avons observé qu'en contexte de constructions référentielles, i. e. dont l'agent n'est pas mis en arrière-plan, les verbes à accord comme les verbes neutres seraient caractérisés par l'assignation de la fonction de sujet à la place du corps du signeur. Sur le plan sémantique, la marque du sujet encodée par le locus corporel ne correspond pas nécessairement à la première personne.

nt aux mécanismes mettant en arrière-plan l'agent, nous formulons l'hypothèse suivante: l'encodage de la fonction de sujet sur un locus corporel ou un autre emplacement dépendrait de la perspective active ou passive mise en valeur par la proposition. Selon cette hypothèse, le locus corporel peut ainsi marquer la fonction sujet, quel que soit le type de verbe employé, quel que soit le rôle thématique qui lui est associé, et quel que soit le degré de référentialité de cet argument.

Qu'en est-il à présent de ce qui distingue les constructions personnelles prototypiques de celles qui illustrent un mécanisme de mise en arrière-plan de l'agent ? De manière générale, notre comparaison de ces constructions ne nous a amenée à n'observer que 
très peu de différences syntaxiques entre ces structures : c'est le contexte sémantique des énoncés qui nous a permis de percevoir les variations. En effet, la fonction de sujet peut être portée par la place du corps du signeur, de manière indifférenciée avec le type de références, i. e. personnelles aussi bien qu'indistinctes.

Par ailleurs, l'un des intérêts majeurs de notre étude réside dans le questionnement de la perspective active ou passive de ces énoncés mettant en arrière-plan l'agent. Ces réflexions nous ont notamment amenée à nous interroger sur l'assignation des fonctions syntaxiques à la place du corps du signeur et au point de départ du mouvement verbal. S'il est aujourd'hui trop tôt pour se positionner, notre analyse laisse entrevoir qu'en LSF, il pourrait exister une distinction entre constructions passives et constructions impersonnelles.

questionnements soulevés par cette étude serviront de point de départ à une analyse plus vaste. Notamment, grâce à l'étude d'un corpus plus large et varié, nous proposerons des résultats quantitatifs et qualitatifs sur l'usage des procédés de mise en arrière-plan de l'agent recensées ici et des autres formes que nous rencontrerons éventuellement. Au regard des analyses faites sur la mise en arrière-plan de l'agent en LS (Janzen et al., 2001; Guitteny, 2005; Barberà \& Quer, 2013) et de l'importante littérature qu'offre cette thématique dans les études sur les langues vocales, notre analyse de corpus aura pour objectif d'aller vers une description des diverses constructions de mise en arrière-plan de l'agent, dont nous tenterons de proposer une typologie. De plus, notre étude pourra être enrichie par la publication à venir des premiers travaux portant sur la question de la référence impersonnelle humaine en LSF (Garcia et al., 2018a à paraître, 2018b ce numéro).

101 Il reste en effet à mener plus en avant une étude syntaxique et sémantique minutieuse de la LSF visant, notamment, à identifier des tendances dans l'usage de certaines mises en arrière-plan de l'agent. Nous tenterons par exemple de déterminer ce qui différencie des énoncés comme ceux des exemples (14) et (15), que notre intuition nous a amenée à interpréter avec des perspectives différentes, entre l'actif et le passif. Finalement, nous chercherons à répondre à la question suivante: pouvons-nous distinguer des constructions impersonnelles et passives en langue des signes française?

Ces questionnements et recherches participeront à accroître la connaissance sur le fonctionnement grammatical de la LSF. Un travail de description et de mise en exergue des mécanismes de mise en arrière-plan de l'agent tel que celui-ci pourra par ailleurs être employé dans le cadre de problématiques d'enseignement et de traduction, et offrir des outils didactiques permettant de repérer puis d'utiliser consciemment de telles structures. La cohabitation des enjeux qu'implique la description du fonctionnement linguistique de la LSF nécessite d'autant plus d'adapter notre choix terminologique. En effet, comme nous l'avons signalé, s'il est clair que la modalité visuo-gestuelle fait la particularité des langues signées, il nous parait intéressant d'adopter un modèle descriptif qui soit compatible avec les outils conceptuels développés dans le cadre de l'étude des langues vocales (Risler, 2018 ce numéro).

Il nous semble important de conclure ici en insistant sur l'équilibre à trouver entre deux risques qu'impliquent ces choix terminologiques. En effet, il nous faut garder à l'esprit une forme de vigilance face aux concepts développés en linguistique des langues vocales. Il convient pour cela de mettre à l'épreuve les terminologies importées de ces études, afin de ne pas appliquer trop rapidement aux langues signées certains concepts qui pourraient alors se révéler non pertinents. En revanche, laisser de côté les 
concepts élaborés dans l'étude des langues vocales risque d'exclure les langues signées de l'étude des langues du monde et compromet la réalisation d'études comparatives. Dans la poursuite de notre étude, nous participerons à cette démarche de mise à l'épreuve des concepts établis pour les langues vocales, afin d'évaluer leur compatibilité face aux spécificités du système des langues signées. Ceci, en plus d'ouvrir des dialogues interdisciplinaires, favorisera également la communication entre les linguistes eux-mêmes et contribuera à réduire l'isolement des études sur les LS.

\section{BIBLIOGRAPHIE}

Amblard, M. \& É. Voisin (2008) Modélisation de la LSF en vue d'une automatisation, Colloque TALN, 9-13 juin, 2008, Avignon.

Ariel, M. (2001) Accessibility theory : An overview, in Sanders, T., Schilperoord, J. \& W. Spooren (eds.) Text representation : Linguistic and psycholinguistic aspects, Amsterdam: John Benjamins, p. 29-87.

Bahan, B., Kegl, J., Lee, R. G., MacLaughlin, D. \& C. Neidle (2000) The licensing of null arguments in American Sign Language, Linguistic Inquiry, 31, 1, p. 1-27.

Bakker, D. \& A. Siewierska (2007) Another take on the notion subject, in Hannay, M. \& G. Steen (eds.) Structural-Functional Studies in English Grammar. (Studies in language Companion Series, 83), Amsterdam: John Benjamins, p. 141-158.

Barberà, G. \& J. Quer (2013) Impersonal reference in Catalan Sign Language (LSC), in Meurant, L., Sinte, A., van Herreweghe, M. \& M. Vermeerbergen (eds.) Sign Language Research Uses and Practices : Crossing Views on Theoretical and Applied Sign Language Linguistics, Berlin/Boston: De Gruyter Mouton and Ishara Press, p. 237-258 (Sign Language and Deaf Communities Series 1).

Blondel, M. \& D. LE GAC (2007) Entre parenthèses... Y a-t-il une intonation en LSF ? Silexicales, 5, p. 1-16.

Boutet, D., Sallandre, M-A. \& I. Fusellier-Souza, I. (2010) Gestualité humaine et langues des signes : entre continuum et variations, Langage et société, 1, 131, p. 55-74.

Cormier, K. (2014) Pronouns, agreement and classifiers: What sign languages can tell us about linguistic diversity and linguistic universals, UCLWPL 2014, p. 1-12.

Cuxac, C. (1993) Iconicité des Langues des Signes, Faits de langues, 1, p. 47-56.

Cuxac, C. (2000) La Langue des Signes Française (LSF). Les voies de l'iconicité, Faits de Langues, 15-16, Paris : Ophrys.

Cuxac, C. (2003) Langue et langage : un apport critique de la langue des signes française [La langue des signes est-elle une langue ? Petite histoire d'une grande question], Langue française, 137, p. 12-31.

Creissels, D. (2006) Syntaxe générale. Une introduction typologique. vol. 1 : Catégories et constructions, Paris : Hermes Sciences Lavoisier. 
De Langhe O., Guitteny P., Portine H. \& C. Retoré (2003) À propos des structures OSV en Langue des Signes Française, in Berthonneau A.-M. \& G. Dal (eds.) Silexicales, 4, p. 115-130.

Dimroth, C., Andorno, C., Benazzo, S. \& J. Verhagen (2010) Given claims about new topics. How romance and Germanic speakers link changed and maintained information in narrative discourse, Journal of Pragmatics, 42, 12, p. 3328-3344.

Dryer, M. S. (2013) Order of Subject and Verb, in Dryer M.S. \& M. Haspelmath (eds.) The World Atlas of Language Structures Online, Leipzig: Max Planck Institute for Evolutionary Anthropology. (http://wals.info/chapter/82, consulté le 30/08/2017).

Engberg-Pedersen, E. (1993) Space in Danish Sign Language, Hamburg: Signum-Verlag.

Garcia, B. (2010) Sourds, surdité, langue(s) des signes et épistémologie des sciences du langage: problématiques de la scripturisation et modélisation des bas niveaux en Langue des Signes Française (LSF), Thèse d'habilitation à diriger les recherches en Sciences du Langage, Université Paris 8.

Garcia, B. \& E. Martinod (2017) Ancrage perceptif et invariant dans les langues des signes, langues de sourds, in Biardzka, E., Danko, M., Komur-Thilloy G. \& F. Marsac (eds.) Echo des études romanes, vol. XIII, Université de Bohème du Sud de Ceské Budejovice et Institut langues et Littératures romanes, République tchèque, p. 73-88.

Garcia, B. Sallandre, M.-A., L'Huillier, M.-T., (à paraître en 2018), Impersonal human reference in French Sign Language (LSF), in Barberà G. \& P. Cabredo Hofherr (coord.) R-Impersonals in Sign Language, Special Issue of Sign Language \& Linguistics, John Benjamins Publishing Company, $32 \mathrm{p}$. Garcia, B., Sallandre, M.-A., L'Huillier, M-T. \& H. Aksen (2018, ce numéro) La référence impersonnelle humaine en langue des signes française, TIPA. Travaux interdisciplinaires sur la parole et le langage [En ligne], 34.

Guitteny, P. (2005) Passif et inverse en langue des signes, Actes de TALN-RECITAL, 2, p. 321-326. Janzen, T., O'Dea, B. \& B. Shaffer (2001) The Construal of Events: Passives in American Sign Language. Sign Language Studies, 1, 3, p. 281-310.

Johnston, T. (2001) Nouns and Verbs in Australian Sign Language: An Open and Shut Case? Journal of Deaf Studies and Deaf Education, Oxford: Oxford University Press, 6, 4, p. 235-257.

Keenan, E.L. (1976) Towards a universal definition of "subject", in Li C.N. (eds.) Subject and Topic, New-York: Academic Press, p. 303-333.

Kimmelman, V. (2016) Impersonal reference in Russian Sign Language, Communication présentée à Grote Taaldag, February 6, 2016, Utrecht, Netherlands.

Kimmelman, V. (2017) Null arguments, agreement, and classifiers in Russian Sign Language, Colloque FEAST, June 22, 2017, Reykjavík, Iceland.

Lazard, G. (1994) L'actance, Paris: PUF.

Lazard, G. (2009) Qu'est-ce qu'un sujet ? La linguistique, 1, 45, p. 151-158.

Lillo-Martin, D. (1986) Two kinds of null arguments in American Sign Language, Natural Language and Linguistic Theory, 4, p. 415-444.

MacLaughlin, D., Neidle, C., Bahan, B. \& R.G. Lee (2000) Morphological Inflections and Syntactic Representations of Person and Number in ASL, Langage et surdité, 29, p. 73-100.

Meir, I., Padden, C.A., Aronoff, M. \& W. Sandler (2007) Body as subject, Journal of linguistics, 43, 3, p. 531-563. 
Meurant, L. (2008) Le regard en langue des signes. Anaphore en langue des signes française de Belgique (LSFB) : morphologie, syntaxe, énonciation, Presses Universitaires de Namur / Presses Universitaires de Rennes (Rivages Linguistiques).

Miller, C., Dubuisson, C. \& C. Poulin (1999) Constructing and maintaining reference and topic in spatial grammar, in Theoretical Issues in Sign Language Issues, November 12-15, 1998, Washington.

Millet, A. (2004) La langue des signes française (LSF) : une langue iconique et spatiale méconnue, Cahiers de l'APLIUT, XXIII, 2, p. 31-44.

Millet, A. (2006) Le jeu syntaxique des proformes et des espaces dans la cohésion narrative en LSF, Glottopol, Les langues des signes (LS) : recherches sociolinguistiques et linguistiques, 7, p. 96-111.

Millet, A. (2008) L'expression de la quantité définie et indéfinie en LSF, Silexicale, 5, p. 77-89.

Moody, B., Hof, D. \& S. Dumartin (1998) La langue des signes, Dictionnaire bilingue LSF / Français. Paris: IVT édition, p. 159.

Morgenstern, A. (1997) L'enfant sourd énonciateur-signeur : l'auto-désignation chez l'enfant en Langue des Signes Française. LIDIL, 15, p. 119-140.

Morgenstern, A., Caët, S. \& F. Limousin (2016) Pointing and Self-reference in French and French Sign Language. Open Linguistics, 2, 1, De Gruyter Open, p. 47-66.

Padden, C. A. (1988) Interaction of Morphology and Syntax in American Sign Language, New York: Garland Press.

Risler, A. (2002) Point de vue cognitiviste sur les espaces en LSF, LIDIL, 26, p. 45-61.

Risler, A. (2007) Les classes lexicales en LSF envisagées à partir de la fonction adjectivale, Silexicales, 5, p. 103-125.

Risler, A. (2011) Corps réel et positions énonciatives en Langue des Signes Française, Représentations du sens linguistique V, 25-27 mai 2011, Chambéry.

Risler, A. (2013) Expression du déplacement dans les langues signées : comment parler d'espace dans une langue spatiale? Faits de Langue, 42, p. 217-244.

Risler, A. (2016) Parler de soi, parler des autres en Langue des Signes Française. Autopointage et prise de rôle, L'Information grammaticale, 149, p. 45-54.

Risler, A. (2018, ce numéro) Changer de regard et de discours sur la langue des signes française, TIPA. Travaux interdisciplinaires sur la parole et le langage [En ligne], 34.

Sallandre, M-A. (2001) Va-et-vient de l'iconicité en Langue des Signes Française, Acquisition et Interaction en Langue Etrangère, 15, p. 37-59.

Sallandre, M-A. (2003) Les unités du discours en Langue des Signes Française. Tentative de catégorisation dans le cadre d'une grammaire de l'iconicité, Thèse de Doctorat en Sciences du Langage, Université Paris 8.

Schembri, A., Wigglesworth, G., Johnston, T., Leigh, G., Adam, R. \& R. Barker (2002) Issues in the development of the Test Battery for Australian Sign Language (Auslan) Morphology and Syntax, Journal of Deaf Studies and Deaf Education, 7, 1, Oxford University Press, p. 18-40.

Siewierska, A. (2008) Ways of impersonalizing: Pronominal vs. verbal strategies, in GómezGonzález, M., Mackenzie L. \& E.M. Gonzáles Álvarez (eds.) Current Trends in Contrastive Linguistics, Amsterdam: John Benjamins, p. 3-26 (Studies in Functional and Structural Linguistics 60). 
Siewierska, A. (2011), Overlap and complementary in reference impersonals: Man-constructions vs. Third person plural-impersonals in the languages of Europe, in Malchukov, A. \& A. Siewierska (eds.) Impersonal Constructions: A cross-linguistic perspective, Amsterdam: John Benjamins, p. 57-90.

Supalla, T. \& E.L. Newport (1978) How many seats in a chair? The derivation of nouns and verbs in American Sign Language, in Siple P. (eds.) Understanding language through sign language research, New York: Academic Press, p. 91-132.

Talmy, L. (1985) Lexicalization patterns : Semantic structure in lexical form, in Shopen, T. (eds.) Language typology and syntactic description vol. 3: Grammatical categories and the lexicon, Cambridge: Cambridge University Press, p. 57-149.

Tomlin, R.S. (1986) Basic word order. Functional principles, London: Croom Helm.

Yau, S. (1993) Iconicité à deux niveaux : de la forme à la motivation, Faits de langues, 1, p. 57-67.

\section{NOTES}

1. Voir : http://www.irit.fr /LS-COLIN.

2. À ce jour, le corpus Finitestory n'a pas fait l'objet d'une mise en ligne.

\section{RÉSUMÉS}

La présente étude a pour objet de comprendre et décrire le fonctionnement syntaxique de la langue des signes française (LSF), et tout particulièrement, ce qui caractérise dans cette langue l'un des constituants centraux de la proposition: l'argument sujet. Il s'agit d'une recherche préliminaire, basée sur un corpus de données semi-spontanées. Dans un premier temps, nous cherchons à identifier les propriétés de l'argument sujet, dans des constructions syntaxiques basiques à référence personnelle. Les propriétés du sujet prototypique définies par cette étape de notre recherche sont par la suite confrontées à l'analyse de constructions dont l'agent est mis en arrière-plan, i. e. référentiellement faible, voire non-instancié lexicalement. En effet, différentes structures de mise en arrière-plan de l'agent, telles que le passif ou l'impersonnel, ont été relevées dans plusieurs langues signées (Janzen et al., 2001; Guitteny, 2005; Barberà \& Quer, 2013 ; Kimmelman, 2016). Notre étude servira ainsi de base à une recherche plus large, ayant pour objet de distinguer les différents procédés de mise en arrière-plan de l'agent en LSF.

Impersonal constructions are a highly debated issue in the studies of spoken languages, especially regarding the description of the Romance and Slavic languages. Impersonal constructions are linguistically distinguished from personal constructions, $i$. e. verbal forms associated with a referential argument in the subject position. The present study is devoted to impersonal constructions with a non-referential subject, while taking into account a very specific language : French Sign Language (LSF).

While spoken languages are said to be visual-gestural and audio-vocal languages, signed languages are characterized by their exclusive visual-gestural modality, involving different features of their linguistic system, such as iconicity or - what is at issue here - the referential use of space. In sign linguistics, spatialization refers to the signer's ability to introduce the reference 
to an entity linked with a specific location in space. For example, this referential strategy allows him or her to point at a location to refer back to the argument that was linked to it earlier in the discourse (Lillo-Martin, 1986 ; Bahan et al., 2000 ; Meurant, 2008 ; Risler, 2013). Therefore, we aim to study how a loss of referentiality can be expressed in a visual-gestural language.

In the recent past, linguistic descriptions of sign languages have provided evidence of agentdefocusing structures, such as the passive (Janzen et al., 2001 ; Guitteny, 2005) or the impersonal constructions (Barberà \& Quer, 2013 ; Kimmelman, 2016). Hence, on the basis of previous findings on impersonal structures in spoken languages, and taking into account studies describing the syntactic functioning of signed languages, this article analyses personal and impersonal structures in LSF. Different items lead our path of reflexion.

Based on identification features of the subject in spoken languages, we define in this article the properties of the subject in LSF. With regard to the literature, we distinguish primarily two paths for analysing the subject in sign language.

On the one hand, French studies reveal that thematic roles are predominant in the analysis of LSF, whereas the syntactic functions of the phrasal constituents are rarely set out. However, the interpreted sentences illustrate in general the choice of an active perspective: the agent corresponds to the syntactic function of subject. We can conclude from these analyses that, with agreement verbs (Padden, 1988), the starting point of the verbal movement is analysed as marking the agent-subject position.

On the other hand, the study of Meir et al. (2007) offers different analysis possibilities, by assigning the syntactic function of the subject to the signer's body, regardless of its thematic role.

In light of these studies, our article highlights possible interpretations of personal and agentdefocusing constructions in French Sign Language, and raises the question whether the subject function is marked on a specific location or not.

In order to conduct this study, we have chosen to work on empirical data, extracted from semispontaneous and narrative corpora, since they favor referential structures and anaphora. The analysis consists of two stages.

The first part of this study is devoted to the analysis of basic sentences in LSF, in which the personal subject is specified, in order to define the features of the subject in this language. This analysis raises a syntactic specificity of sign language : like other signed languages (Lillo-Martin, 1986 ; Bahan et al., 2000 ; Kimmelman, 2017), LSF accepts the argument position of the subject, and/or the object, to be lexically empty. In the context of this study, we focus on the subject argument and note that the subject position is not systematically filled by a lexical component. Our analysis suggests that the arguments are mentioned by the expression of anaphora, in the predicative form : the syntactic mark of the subject argument on a spatial location allows the spatial instantiation of the argument in the inflection. Moreover, the observation of personal references allows us to note the role the body plays in the argumental structure, encoding the properties of the subject argument. Following Meir et al. (2007), we analyse this corporal locus as marking the syntactical function of the subject. However, we suggest that the syntactic function of the subject is marked by the corporal locus, irrespective of the type of the verb.

The second part of this study is devoted to structures with an unspecified human subject and compares them to the personal constructions analysed in the first part. The comparative approach does not show syntactic distinctions between these personal and impersonal structures. Indeed, we observe both lexical and empty subject constructions with personal as with non-specified subjects. Finally, with respect to the role of the body, this comparative study allows us to observe that the body encodes the properties of the first argument regardless of its referentiality. Then, in the absence of syntactic differences between personal and impersonal references, only the semantic level allows us to notice the distinction. 
In personal as well as in agent-demoting constructions, this assignation of the subject function to the body calls into question the interpretation of the starting point of agreement verbs' movement. Indeed, if the end point of the verbal movement - which represents the patient - is on the signer's body, and is assigned the function of subject, it may consequently be a structure defocusing the agent. This assumption is corroborated by the observation of the use of specific spatial markings, on a high and lateral space, emphasizing the process by not referring to any specified agent. Similar spatial markings have been studied for Catalan Sign Language (LSC) (Barberà \& Quer, 2013), giving us an interesting perspective to guide our analysis of this phenomenon. Moreover, previous French studies on the role of space in the actantial distribution in LSF describe the use of a high lateral space, on each side of the signer's head, for the reference to an indefinite agent (Millet, 2004, 2006).

These considerations in relation to the assignation of the subject syntactical function to the signer's body or to the starting point of the verbal movement led us in our questioning of the passive or the active perspective. Though it is too soon to settle the question, this analysis suggests that a distinction between impersonal and passive constructions could be drawn. Thus, the questioning raised by this study will be the basis for further research on defocusing agent strategies and their semantic tendencies in LSF, with the aim of distinguishing impersonal and passive constructions in this language.

INDEX

Keywords : sign language, syntax, subject argument, impersonal, spatialization

Mots-clés : langue des signes, syntaxe, argument sujet, impersonnel, spatialisation

\section{AUTEUR}

\section{MYRIAM CHARPENTIER}

Université de Lille, CNRS, Savoirs Textes Langage, Lille, France

Université de Namur, LSFB-Lab, NaLTT, Namur, Belgique.

myriam.charpentier@univ-lille.fr 